\title{
REGLAS DE COMBINACIÓN DE LOS EFECTOS DE LAS TRES COMPONENTES DE TERREMOTOS Y RESPUESTA CRÍTICA
}

\author{
Federico Valenzuela Beltrán ${ }^{(1)}$, Alfredo Reyes Salazar ${ }^{(1)}$, David De León Escobedo ${ }^{(2)}$, \\ Edén Bojórquez Mora ${ }^{(I)}$ y Arturo López Barraza ${ }^{(I)}$
}

\begin{abstract}
RESUMEN
La precisión de las reglas de combinación del 30\% y SRSS, y la orientación crítica de las componentes de terremotos se estudian en este trabajo. Se analizan modelos estructurales complejos que representan edificios de baja y mediana altura. Se realiza un análisis estadístico de la precisión de las reglas de combinación aplicadas a parámetros de respuesta individuales y múltiples. El efecto de la correlación entre las componentes de los terremotos en dicha precisión también se estudia. Finalmente, se realizan análisis con varios ángulos de incidencia de las componentes con la finalidad de encontrar la orientación crítica. Los resultados muestran que las reglas subestiman la carga axial en columnas, pero sobreestiman razonablemente los cortantes de entrepiso. Ambas reglas son más conservadoras cuando los modelos se excitan por las tres componentes. Los efectos individuales pueden estar altamente correlacionados, incluso para componentes principales no correlacionadas. Las reglas no siempre son precisas para valores pequeños de coeficientes de correlación, y valores altos de éstos no siempre están relacionados a una estimación imprecisa de la respuesta combinada. La precisión de las reglas de combinación depende del grado de correlación de las componentes, del parámetro de respuesta, de la localización del elemento considerado y del nivel de deformación estructural.
\end{abstract}

Palabras clave: códigos de diseño sísmico; edificios de acero; reglas de combinación; correlación de los efectos individuales; respuesta crítica

\section{COMBINATION RULES OF THE EFFECTS OF THREE COMPONENTS OF EARTHQUAKES AND CRITICAL RESPONSE}

\begin{abstract}
The accuracy of the $30 \%$ and SRSS rules, the critical orientation of the components of earthquakes, and some related issues, are studied in this work. Complex structural models representing low and medium height buildings are analyzed. A statistical analysis of the precision of the combination rules, applied to individual and multiple response parameters, is performed. The effect of the correlation of the components of earthquakes in such precision is also studied. Finally, several analyses with various incidence angles of the components are performed with the purpose of finding the critical orientation. Results show that the rules underestimate the axial load in columns, but reasonably overestimate the interstory shears. Both rules are more conservative when the models
\end{abstract}

\footnotetext{
Artículo recibido el 24 de febrero de 2014 y aprobado para su publicación el 20 de agosto de 2015. Se aceptarán comentarios y/o discusiones hasta cinco meses después de su publicación.

(1) Facultad de Ingeniería, Universidad Autónoma de Sinaloa, Calzada de las Américas y Boulevard Universitarios S/N, Ciudad Universitaria, Culiacán, Sinaloa, México, C.P. 80040. f.valenzuelab@hotmail.com, reyes@uas.edu.mx, eden@uas.edu.mx alopezb@uas.edu.mx

${ }^{(2)}$ Facultad de Ingeniería, Universidad Autónoma del Estado de México, Ciudad Universitaria, Toluca, Estado de México. daviddeleonescobedo@yahoo.com.mx
} 
are excited by the three components. The individual effects may be highly correlated, even for principal components. The rules are not always accurate for small values of correlation coefficients, and high values of such coefficients are not always related to an inaccurate estimation of the combined response. The accuracy of the combination rules depends on the degree of correlation of the components, the response parameter, the location of the considered member and the level of structural deformation.

Keywords: seismic design codes; steel buildings; combination rules; correlation of individual effects; critical response

\section{INTRODUCCIÓN}

La ocurrencia de sismos fuertes en diversas partes del mundo ha producido daños materiales significativos y un gran número de pérdidas de vidas humanas. Por lo anterior, los procedimientos de análisis y diseño sísmico de estructuras establecidos en los códigos se modifican periódicamente. Se han propuesto varios métodos en muchos códigos, como el Método Lateral Equivalente y varios métodos de análisis dinámico (análisis modal, espectral, análisis paso a paso lineal y análisis paso a paso no lineal). El Reglamento de Construcciones del Distrito Federal (RCDF, 2004), en el apartado "Normas Técnicas Complementarias para Diseño por Sismo", considera los métodos anteriores e identifica los requisitos mínimos para la aplicación de un método particular. El International Building Code (IBC, 2009) también considera estos procedimientos así como también los requerimientos para su aplicación.

Nuestro entendimiento del fenómeno sísmico ha mejorado significativamente en los últimos años. Este avance debe ser ubicado en el contexto de estimaciones más precisas de la respuesta de estructuras, ya que es un aspecto fundamental para el diseño apropiado de las mismas. Debido a los avances en la tecnología informática, la capacidad de cómputo se ha incrementado significativamente en los últimos años. Ahora es posible estimar el comportamiento en el dominio del tiempo de estructuras modeladas en tres dimensiones ante la acción de cargas sísmicas en una forma muy realista, lo que permite a la vez estudiar la exactitud de los métodos simplificados. El conocer las limitaciones de los métodos simplificados permitirá mejorar nuestra comprensión del problema y diseñar estructuras más resistentes ante la acción de cargas sísmicas intensas.

Para fines de análisis de la respuesta estructural, la energía liberada durante un terremoto se expresa en la forma de tres registros de aceleración: dos horizontales y uno vertical. Las excitaciones rotacionales no se miden y se ignoran completamente en el análisis. Además, para movimientos de fuente lejana, el efecto de la componente vertical es usualmente menor que el de las componentes horizontales y por ello a menudo se desprecia. Otra razón para despreciar el efecto de la componente vertical es que los edificios se diseñan para cargas de gravedad lo que provee un alto factor de seguridad en la dirección vertical (Newmark y Hall, 1982; Salmon et. al. 2009). Por lo tanto, cuando una estructura se analiza, dos componentes horizontales se aplican generalmente en dirección de sus dos ejes principales asumiendo implícitamente que producirán la máxima respuesta. La precisión de esta práctica y otros aspectos constituye, como se discutirá más adelante, uno de los objetivos de esta investigación.

En los procedimientos rutinarios de análisis simplificados, después de considerar cada una de las componentes sísmicas en forma separada, sus efectos se combinan de diferentes maneras. Este concepto ha sido implementado en muchos códigos. Los procedimientos comúnmente utilizados son la regla del 30 por ciento (30\%) y la de la Raíz Cuadrada de la Suma de los Cuadrados (SRSS). Los códigos, sin embargo, no establecen explícitamente la aplicabilidad de estas reglas: no se especifica la forma de seleccionar la 
orientación crítica de las componentes ortogonales ni el tipo de estructuras (sistemas simples o complejos) a ser considerados, o si las reglas se pueden aplicar a comportamiento estructural elástico o inelástico. No se especifica tampoco si las respuestas individuales producidas por cada componente deben ser colineales (por ejemplo, carga axial en columnas) o no-colineales (por ejemplo, cortante basal), locales (por ejemplo, momento en columnas) o globales (por ejemplo, desplazamientos de entrepiso o de azotea), o si las reglas deben ser aplicadas a un parámetro de respuesta individual o a varios simultáneamente. Además, las reglas generalmente se formulan en los códigos para los efectos de las dos componentes horizontales y suponen implícitamente que dichas componentes y sus correspondientes efectos no están correlacionados. La precisión de estas reglas de combinación, desarrolladas esencialmente para procedimientos de análisis modal lineal, se estudia en esta investigación para determinar su aplicabilidad considerando los aspectos antes mencionados. El estudio se realiza primero para las dos componentes horizontales y después para las tres componentes considerando explícitamente la correlación de las componentes y la de sus efectos individuales en la respuesta estructural.

\section{REVISIÓN DE LITERATURA Y OBJETIVOS}

La orientación crítica de las componentes de un terremoto y las formas de combinar sus efectos individuales, han sido tópicos de interés para la ingeniería sísmica durante las últimas décadas. Penzien y Watabe (1975) plantearon que las tres componentes de un terremoto no están correlacionadas a lo largo de los ejes principales y que el eje principal mayor es horizontal y dirigido hacia el epicentro, el eje intermedio es horizontal y perpendicular a la orientación de la dirección principal, y el eje principal menor es vertical. La respuesta crítica se obtendría cuando estas componentes se aplican en dirección de los ejes estructurales. Rosenblueth (1980) estableció "La ausencia de correlación de los acelerogramas principales asegura que las respuestas también son no correlacionadas". Smeby y Der Kiureghian (1985) observaron que, para análisis espectral de estructuras lineales, el efecto de la correlación entre las componentes de terremotos es relativamente pequeño y es insignificante cuando las componentes a lo largo de los ejes principales estructurales tienen intensidades idénticas o casi idénticas. Newmark (1975), Rosenblueth y Contreras (1977) y Newmark y Hall (1982) propusieron la regla del porcentaje para aproximar la respuesta combinada como la suma del $100 \%$ de la respuesta resultante de una componente y un porcentaje $(\lambda)$ de las respuestas producidas por las otras dos componentes. Para combinar los efectos de las dos componentes horizontales, Newmark (1975) sugirió un valor de $40 \%$ para $\lambda$ y Rosenblueth y Contreras (1977) propusieron un valor de 30\%. Se han reportado muchos otros estudios en relación a la combinación de las respuestas sísmicas producidas por dos o tres componentes. Utilizando análisis elásticos y una estructura simple en tres dimensiones, Wilson et al. (1995) observaron que la regla de combinación del porcentaje podría subestimar las fuerzas de diseño en algunos de los miembros. López et al. (2000) propusieron una fórmula para calcular el valor crítico de las respuestas estructurales producidas por las componentes principales horizontales actuando a lo largo de cualquier ángulo de incidencia con respecto a los ejes estructurales. Heredia y Machicao (2004a, 2004b) estudiaron los efectos de las componentes horizontales ortogonales utilizando un modelo estructural elástico de un nivel torsionalmente rígido primero, y torsionalmente flexible después, para el caso de suelos blandos y firmes. Encontraron que, en general, para suelos firmes los efectos son importantes para sistemas torsionalmente flexibles y traslacionalmente rígidos y que la regla de combinación del porcentaje puede sobreestimar o subestimar la respuesta estructural, particularmente para suelos torsionalmente flexibles. López et al. (2006) investigaron las características de los espectros de respuesta de las componentes principales y determinaron las relaciones entre dichos espectros. Beyer y Bommer (2007) estudiaron varios aspectos involucrados en la selección y escalamiento de registros sísmicos para análisis bi-direccional. Demostraron que la respuesta estructural varía en función del ángulo de incidencia de las componentes de los sismos con respecto a los ejes estructurales y que la mediana de la respuesta para todos los ángulos posibles podría ser el parámetro más adecuado. Rigato y Medina (2007) examinaron el efecto que tiene el 
ángulo de incidencia en una estructura de una sola planta sometida a movimientos bi-direccionales. Demostraron que la aplicación simultánea de las componentes a lo largo de los ejes principales de la estructura podría subestimar las demandas inelásticas máximas. Más recientemente, Valdez y Ordaz (2008) desarrollaron algunas expresiones analíticas para estimar la respuesta máxima combinada de estructuras elásticas producida por las dos componentes horizontales de terremotos registrados en suelo blando usando la teoría de vibraciones aleatorias. McKenna y Feneves (2009) investigaron los efectos de la dirección de aplicación de la fuerza sísmica en las respuestas de puentes asimétricos tipo viga-losa usando espectros de respuesta y análisis paso a paso. Se examinaron las reglas de combinación del porcentaje $30 \%$ y $40 \%$, así como la regla SRSS. Se concluyó que tanto la regla SRSS como la del porcentaje del $40 \%$ podrían utilizarse en el caso de análisis espectral de la respuesta de puentes asimétricos. Para los análisis paso a paso, sin embargo, ninguna de las reglas proporcionó resultados conservadores. Recomendaron la aplicación de las dos componentes de aceleración para varías direcciones angulares. Bisadi y Head (2010) investigaron los efectos ortogonales en el análisis no lineal de puentes de un solo claro sometidos a la acción de dos componentes. Demostraron que el ángulo crítico de excitación no es el mismo en los modelos lineales y no lineales y que el procedimiento de la AASTHO para estimar los efectos individuales de las componentes puede subestimar la respuesta máxima probable. Mackie y Cronin (2011) estudiaron el efecto del ángulo de incidencia de las componentes de excitación en la respuesta de puentes de autopistas. Calcularon las medias de los espectros de respuesta elásticos e inelásticos para las estructuras modeladas como sistemas de un grado de libertad usando diversas técnicas de orientación. Encontraron que el ángulo de incidencia tiene un efecto despreciable sobre las medias de las respuestas.

A pesar de las importantes contribuciones de los estudios anteriores sobre las reglas de combinación, la mayoría de ellos se limitaron a análisis elástico aplicado a estructuras modeladas como sistemas de un grado de libertad o a estructuras de unos pocos pisos conectados con diafragmas rígidos. El comportamiento inelástico de los elementos estructurales existentes en sistemas complejos, como edificios de varios niveles, y los mecanismos de disipación de energía que en ellos se presentan no han sido explícitamente considerados. Reyes-Salazar et al. (2000), Reyes Salazar y Haldar (1999, 2000, 2001a, 2001b) y Bojórquez et al. (2010) mostraron que la energía disipada tiene un efecto muy importante en la respuesta estructural de edificios de acero con marcos resistentes a momento. Más recientemente, ReyesSalazar et al. (2004, 2008), mediante análisis paso a paso no lineales de sistemas complejos de varios grados de libertad, observaron que tanto la regla del 30\% como la SRSS pueden subestimar la respuesta combinada para el caso de las componentes horizontales y que los mecanismos de disipación de energía se deben considerar de la forma más precisa posible. Sin embargo, en estos estudios se usaron sistemas estructurales hipotéticos y no se consideró el efecto de la correlación de las componentes de los terremotos en la precisión de las reglas ni parámetros múltiples de respuesta. Además, solo se usaron las dos componentes horizontales.

Los objetivos principales de esta investigación son: a) estudiar la precisión de las reglas de combinación comúnmente usadas, para sistemas complejos de varios grados de libertad (SVGL), para comportamiento elástico e inelástico, para parámetros de respuesta individuales y múltiples, considerando explícitamente la correlación de las componentes y la de sus correspondientes efectos, para las componentes normalmente registradas (denotadas en lo sucesivo como componentes normales) y para componentes no correlacionadas (principales); b) obtener la orientación crítica de las componentes ortogonales para parámetros de respuesta colineales y no colineales considerando varios ángulos de incidencia. Para lograr los objetivos mencionados, se estimaron las respuestas elásticas e inelásticas de algunos modelos estructurales, los cuales se excitaron por veinte registros sísmicos en el dominio del tiempo. Se considera el caso particular de edificios de acero con marcos resistentes a momento perimetrales. 


\section{METODOLOGÍA}

\section{Modelo matemático}

Para lograr los objetivos planteados en este estudio, es necesario evaluar de la manera más realista posible la respuesta sísmica elástica e inelástica de edificios de acero modelados como SVGL sometidos a la acción de terremotos intensos. Para ello, se usará un algoritmo basado en el Método del Elemento Finito y en hipótesis de esfuerzos. Dicho algoritmo ha sido automatizado a través de un programa de cómputo. El programa (Reyes-Salazar, 1997) puede estimar la respuesta sísmica no lineal de marcos de acero modelados como SVGL considerando las no linealidades geométrica y del material. Además, es muy eficiente en comparación con la formulación basada en hipótesis de desplazamientos comúnmente usada. La respuesta estructural en términos de fuerzas de miembro (fuerzas axial y cortante, y momentos flexionantes y torsionantes), cortante total en la base, desplazamientos de entrepiso o cualquier otro parámetro de respuesta, se puede estimar usando dicho programa. Los resultados de este programa han sido verificados con resultados teóricos y experimentales (Reyes-Salazar y Haldar 2000, Reyes-Salazar y Haldar 2001b). Los detalles de la teoría de esta aproximación están fuera del alcance de este estudio. Sin embargo, éstos se pueden encontrar en la literatura (Kondo y Atluri, 1987).

\section{Modelos estructurales}

Tabla 1. Secciones de vigas y columnas para los Modelos 1 y 2

\begin{tabular}{|c|c|c|c|c|c|c|c|}
\hline \multirow{3}{*}{ Modelo } & \multirow{3}{*}{ Nivel } & \multicolumn{3}{|c|}{ Marcos Resistentes a Momento } & \multicolumn{3}{|c|}{ Marcos de Gravedad } \\
\hline & & \multicolumn{2}{|c|}{ Columnas } & \multirow{2}{*}{ Vigas } & \multicolumn{2}{|c|}{ Columnas } & \multirow{2}{*}{ Vigas } \\
\hline & & Exterior & Interior & & Exterior & Interior & \\
\hline \multirow{3}{*}{1} & $1 / 2$ & $\mathrm{~W} 14 \times 257$ & $\mathrm{~W} 14 \times 311$ & W33x118 & W14x82 & W14x68 & W18x35 \\
\hline & $2 / 3$ & W14x257 & $\mathrm{W} 14 \times 312$ & W30x116 & W14x82 & W14x68 & W18x35 \\
\hline & 3/Azotea & $\mathrm{W} 14 \times 257$ & $\mathrm{~W} 14 \times 313$ & W24x68 & W14x82 & W14x68 & W16x26 \\
\hline \multirow{10}{*}{2} & $-1 / 1$ & W14x370 & W14x500 & W36x160 & W14x211 & W14x193 & W18x44 \\
\hline & $1 / 2$ & W14x370 & W14x500 & W36x 160 & W14x211 & W14x193 & W18x35 \\
\hline & $2 / 3$ & W14x370 & W14x500,W14x455 & W36x160 & W14x211,W14x159 & W14x193,W14x145 & W18x35 \\
\hline & $3 / 4$ & W14x370 & W14x455 & W36x135 & W14x159 & W14x145 & W18x35 \\
\hline & $4 / 5$ & $\mathrm{~W} 14 \times 370, \mathrm{~W} 14 \times 283$ & $\mathrm{~W} 14 \times 455, \mathrm{~W} 14 \times 370$ & W36x135 & W14x159,W14x120 & W14x145,W14x109 & $\mathrm{W} 18 \times 35$ \\
\hline & $5 / 6$ & W14x283 & W14x370 & W36x135 & W14x120 & W14x109 & W18x35 \\
\hline & $6 / 7$ & W14x283,W14x257 & W14x370,W14x283 & W36x135 & W14x120,W14x90 & W14x109,W14x82 & W18x35 \\
\hline & $7 / 8$ & W14x257 & W14x283 & W30x99 & W14x90 & W14x82 & W18x35 \\
\hline & $8 / 9$ & W14x257,W14x233 & W14x283,W14x257 & W27x84 & W14x90,W14x61 & W14x82,W14x48 & W18x35 \\
\hline & 9/Azotea & $\mathrm{W} 14 \times 233$ & $\mathrm{~W} 14 \times 257$ & $\mathrm{~W} 24 \times 68$ & W14x61 & $\mathrm{W} 14 \times 48$ & $\mathrm{~W} 16 \times 26$ \\
\hline
\end{tabular}

Como parte del proyecto de la SAC (Structural Engineers Association of California, Applied Technology Council and California Universities for Research in Earthquake Engineering), se comisionó a tres prestigiadas firmas de consultoría de Estados Unidos por The Federal Emergency Management Agency (FEMA, 2000) para realizar el diseño de varios modelos de edificios de acero con marcos resistentes a momento (MRM) perimetrales y marcos de gravedad (MG) en el interior. Los modelos son de 3, 9 y 20 niveles y se diseñaron de acuerdo a los códigos de construcción de las siguientes tres ciudades: Los Ángeles (UBC, 1994), Seattle (UBC, 1994) y Boston (BOCA, 1993). Los modelos de 3 y 9 
niveles, que representan la zona de Los Ángeles y los diseños pre-Northridge, se usan en esta investigación para estudiar los problemas mencionados anteriormente. Se denotan en lo sucesivo como Modelos 1 y 2, respectivamente. Las dimensiones en planta y elevación mostrando la localización de los marcos resistentes a momento (líneas continuas), y los miembros estructurales (columnas) considerados en el estudio se muestran en la Figura 1. Las secciones de vigas y columnas de los modelos se dan en la Tabla 1.

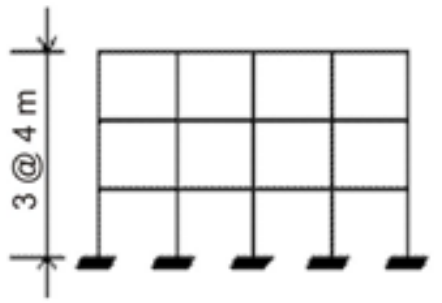

a) Elevación Modelo 1

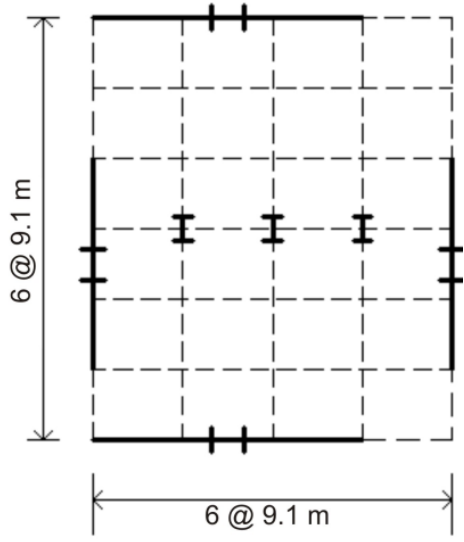

b) Planta Modelo 1

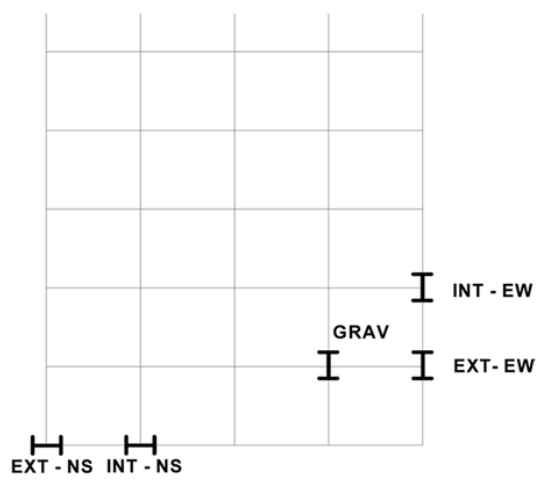

c) Elementos estudiados Modelo 1

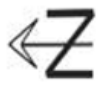

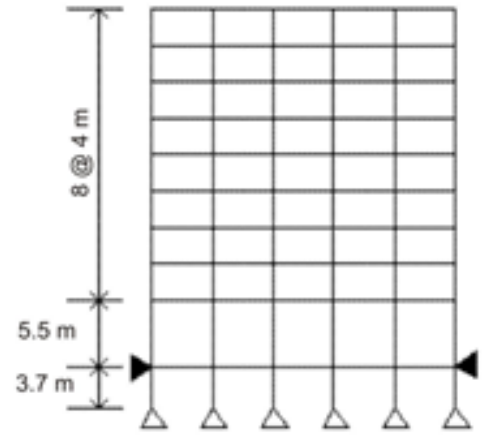

d) Elevación Modelo 2

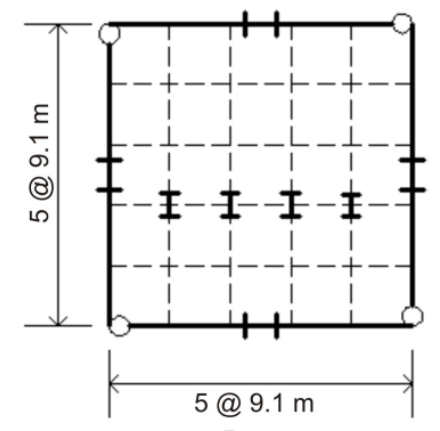

e) Planta Modelo 2

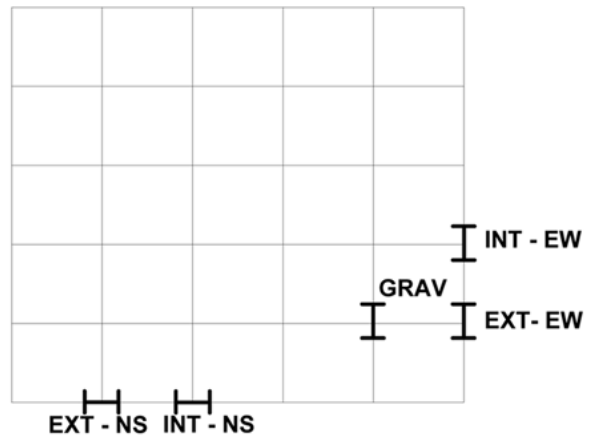

f) Elementos estudiados Modelo 2

Figura 1. Elevación, planta y elementos estudiados de los Modelos 1 y 2

Los periodos fundamentales de los Modelos 1 y 2 son de 1.03 y 2.34 segundos, respectivamente, representando edificios de baja y mediana altura. El amortiguamiento considerado es de 5\% del amortiguamiento crítico, el mismo que se utiliza comúnmente en los reglamentos. Las columnas de los MRM perimetrales del Modelo 1 se consideran empotradas en la base mientras que las del Modelo 2 están articuladas. En todos los marcos las columnas son de acero Grado 50 y las vigas de acero A36. Para los dos modelos las columnas de gravedad se consideran articuladas en la base. Todas las columnas de los MRM perimetrales se flexionan alrededor del eje fuerte. El eje fuerte de las columnas de gravedad está orientado en la dirección N-S. El diseño de los MRM en las dos direcciones ortogonales es prácticamente el mismo. Para los propósitos de esta investigación se seleccionaron estructuras de acero, pero igualmente se podrían haber usado estructuras de concreto. Sin embargo, se usan edificios de acero ya que la disipación de energía tiene un mayor efecto en la respuesta sísmica que en edificios de concreto. Además 
los modelos aquí utilizados fueron sugeridos por FEMA y han sido usados por muchos investigadores para estudiar el comportamiento sísmico de estructuras de acero con marcos resistentes a momento perimetrales y se usan aquí también como modelos de referencia. En el análisis sísmico tridimensional de edificios, usualmente se consideran tres grados de libertad por nivel; un desplazamiento rotacional y dos traslacionales. En este estudio los marcos se modelan como SVGL, cada columna se representa por un elemento y cada viga se representa por dos elementos, con un nudo en el medio. Se considera que todos los nudos tienen seis grados de libertad. El número total de grados de libertad es de 846 y 3408 para los Modelos 1 y 2, respectivamente.

\section{Acciones sísmicas}

La respuesta dinámica de una estructura sometida a la acción de diferentes registros sísmicos, aun cuando se normalicen con respecto a su máxima aceleración o en términos de la pseudo-aceleración evaluada en el periodo fundamental de la estructura $\left(\mathrm{S}_{\mathrm{a}}\left(\mathrm{T}_{1}\right)\right)$, serán en general diferentes para cada registro, reflejando su distinto contenido de frecuencias. Así que, la evaluación de la respuesta estructural usando un solo registro sísmico puede no representar el comportamiento real. En el presente trabajo, los modelos se excitan por veinte registros sísmicos en el dominio del tiempo con diferente contenido de frecuencias. Inicialmente, con el fin de obtener un comportamiento elástico y una demanda sísmica similar para cada terremoto, los registros utilizados se escalan (generalmente hacia abajo) en términos de la pseudo-aceleración evaluada en el modo fundamental de vibración de la estructura $\left(S_{\mathrm{a}}\left(\mathrm{T}_{1}\right)\right)$. En otras palabras, para un modelo dado, los registros se escalan de tal manera que los valores del espectro de respuesta de pseudo-aceleración evaluado en el período fundamental $\left(\mathrm{T}_{1}\right)$ del modelo, sea el mismo para todos los registros (se tomó como referencia la componente horizontal en la dirección N-S). Después, partiendo de los registros ya escalados como se mencionó, para obtener un comportamiento inelástico, los terremotos se escalan uniformemente hacia arriba de tal manera que para el terremoto crítico los modelos desarrollan un nivel de deformación cercano a un mecanismo de colapso. Obviamente, el desplazamiento de entrepiso varió de un terremoto a otro (de $2 \%$ a 3\%). Debe tenerse en cuenta que los factores de escala son diferentes para cada modelo estructural ya que sus períodos fundamentales son también diferentes.

Las características de los registros sísmicos se muestran en la Tabla 2. Como se puede observar en la tabla, los periodos dominantes de los terremotos varían de 0.12 a $0.88 \mathrm{~s}$. El periodo dominante para cada sismo se define como el periodo donde ocurre el valor máximo de la pseudo-aceleración en el espectro de respuesta elástico. Es importante mencionar que todos los registros seleccionados corresponden a suelo duro e intermedio. Los registros se obtuvieron de diferentes fuentes de información entre los que se mencionan el Conjunto de Datos del Programa Nacional de Movimientos Fuertes (NSMP por sus siglas en inglés) del Banco de Datos Geológicos de Estados Unidos (USGS por sus siglas en inglés). Se puede obtener información adicional acerca de estos sismos de dicha base de datos.

Tabla 2. Características de los registros sísmicos

\begin{tabular}{ccccccc}
\hline Sismo & Localización de la estación & Fecha & T (seg) & $\begin{array}{c}\text { Epicentro } \\
(\mathrm{km})\end{array}$ & Profundidad (km) & $\begin{array}{c}\text { PGA } \\
\left(\mathrm{cm} / \mathrm{s}^{2}\right)\end{array}$ \\
\hline 1 & Cerro Prieto, Baja California, México & $09 / 06 / 1980$ & 0.12 & 20 & 4.6 & 308 \\
2 & Lake Mathews Dam, California & $02 / 09 / 2007$ & 0.15 & 13 & 12.5 & 507 \\
3 & Salton Sea Wildlife Refuge, California & $02 / 09 / 2005$ & 0.19 & 2 & 9.70 & 236 \\
4 & Bear Valley, Webb Residence, California & $27 / 08 / 2011$ & 0.21 & 13 & 7.60 & 239 \\
5 & Paicines, Hain Homestead, California & $06 / 04 / 2012$ & 0.23 & 3.8 & 5.60 & 232 \\
\hline
\end{tabular}


Tabla 2. Características de los registros sísmicos (continuación)

\begin{tabular}{ccccccc}
\hline Sismo & Localización de la estación & Fecha & T (s) & $\begin{array}{c}\text { Epicentro } \\
(\mathrm{km})\end{array}$ & Profundidad (km) & $\begin{array}{c}\text { PGA } \\
\left(\mathrm{cm} / \mathrm{s}^{2}\right)\end{array}$ \\
\hline 6 & Parkfield, Eades, California & $28 / 09 / 2004$ & 0.24 & 9.8 & 7.9 & 384 \\
7 & Redlands, Seven Oaks Dam, California & $16 / 06 / 2005$ & 0.25 & 10 & 11.80 & 290 \\
8 & Holtville. Baja California, México & $30 / 12 / 2009$ & 0.26 & 42.2 & 6.00 & 322 \\
9 & Granada Hills, Porter Ranch, California & $09 / 08 / 2007$ & 0.27 & 6 & 7.50 & 148 \\
10 & Compton, Cressey Park, California & $18 / 05 / 2009$ & 0.3 & 9.9 & 15.10 & 207 \\
11 & Mountain Center, PineMeadows R., California & $12 / 06 / 2005$ & 0.31 & 5.1 & 14.10 & 200 \\
12 & Cobb, California & $18 / 02 / 2004$ & 0.32 & 1.2 & 3.60 & 213 \\
13 & San Jose, Private Residence, California & $31 / 10 / 2007$ & 0.35 & 10.8 & 9.20 & 199 \\
14 & Martinez, VA Medical Clinic, California & $02 / 03 / 2007$ & 0.39 & 10.5 & 16.60 & 149 \\
15 & San Luis Obispo, Rec. Center, México & $22 / 12 / 2003$ & 0.4 & 61.5 & 7.60 & 162 \\
16 & Calexico Fire Station, México & $04 / 04 / 2010$ & 0.4 & 62.6 & 10.00 & 266 \\
17 & Mountain Center, PineMeadows R., California & $07 / 07 / 2010$ & 0.75 & 20.4 & 11.7 & 185 \\
18 & Morongo Valley Fire Station, California & $28 / 06 / 1992$ & 0.81 & 28.8 & 5.00 & 198 \\
19 & Olympia, WDOT Highway Test Lab, Washington & $28 / 02 / 2001$ & 0.82 & 18.3 & 59 & 21.7 \\
20 & Ferndale, Lost Coast Ranch, California & $10 / 01 / 2010$ & 0.88 & 36.4 & 352 \\
\hline
\end{tabular}

\section{REGLAS DE COMBINACIÓN Y CASOS DE CARGA}

\section{Reglas de combinación}

Las reglas de combinación se definen formalmente a continuación. Para efectos de facilitar la discusión, $R_{X}$ representará el efecto máximo absoluto en un punto determinado cuando la estructura se excita por la componente horizontal aplicada en la dirección $N$-S. De igual manera, $R_{Y}$ y $R_{Z}$ representarán el efecto máximo correspondiente cuando la estructura se excita por las componentes en dirección $E$ - $W$ y vertical, respectivamente. Los efectos producidos por cada componente se pueden calcular utilizando varios métodos incluyendo el método lateral equivalente, análisis modal y análisis paso a paso. Para el caso de análisis paso a paso, el comportamiento estructural podría ser elástico o inelástico dependiendo de la intensidad de la excitación, de las características dinámicas de la estructura y del terremoto en cuestión. Usando la regla del porcentaje, el efecto combinado de las tres componentes se puede calcular como la más crítica de las siguientes tres combinaciones:

$R_{C 1}=R_{X}+\lambda R_{Y}+\lambda R_{Z}$

$R_{C 1}=\lambda R_{X}+R_{Y}+\lambda R_{Z}$

$R_{C 1}=\lambda R_{X}+\lambda R_{Y}+R_{Z}$

Un valor de $\lambda=0.3$ representa la regla del $30 \%$ para las tres componentes. De acuerdo con la regla SRSS la respuesta combinada se puede estimar como: 
$R_{C 2}=\sqrt{R_{X}^{2}+R_{Y}^{2}+R_{Z}^{2}}$

Como se mencionó anteriormente, en la mayoría de los códigos, las reglas de combinación se especifican para combinar los efectos únicamente de las dos componentes horizontales. En este caso particular las ecuaciones (1) y (2) se expresan como:

$R_{C 1}=R_{X}+\lambda R_{Y}$

$R_{C 1}=\lambda R_{X}+R_{Y}$

$R_{C 2}=\sqrt{R_{X}^{2}+R_{Y}^{2}}$

La hipótesis básica de la regla SRSS aplicada a las dos componentes horizontales es que no existe correlación entre ellas ni entre sus efectos. La validez de esta hipótesis será evaluada con los resultados de este estudio.

\section{Casos de carga}

Para estudiar la precisión de las reglas de combinación se requiere estimar la respuesta estructural de la manera más precisa posible (también llamada respuesta de referencia) de ambos modelos cuando se excitan simultáneamente por las componentes normales (o principales) de los terremotos. Para facilitar la discusión, se usará la siguiente notación en el resto del artículo. Como se mencionó en la sección "Modelos estructurales", los ejes estructurales se denotan como N-S (horizontal) y E-W (horizontal) y V (vertical). Si consideramos las tres componentes de un terremoto, la primera componente horizontal se denotará por la letra X, la segunda componente horizontal por la letra Y, y la componente vertical por la letra $\mathrm{Z}$. Los símbolos $\mathrm{X}_{\mathrm{n}}, \mathrm{Y}_{\mathrm{n}} \mathrm{y} \mathrm{Z}_{\mathrm{n}}$ indicarán que las estructuras se excitan por las componentes normales; por otra parte los símbolos $\mathrm{X}_{\mathrm{p}}, \mathrm{Y}_{\mathrm{p}}$ y $\mathrm{Z}_{\mathrm{p}}$ indicarán que se aplican las componentes principales. Por lo tanto la notación $\left(X_{p}, Y_{p}, Z_{p}\right)$ indica que las estructuras se excitan simultáneamente por la primera componente horizontal principal, la segunda componente horizontal principal y la vertical principal, en las direcciones $\mathrm{N}-\mathrm{S}, \mathrm{E}-\mathrm{W}, \mathrm{y} \mathrm{V}$ de la estructura, respectivamente. En otras palabras, el primero, segundo, y tercer elemento del vector anterior (ejemplo $\left(\mathrm{X}_{\mathrm{p}}, \mathrm{Y}_{\mathrm{p}}, \mathrm{Z}_{\mathrm{p}}\right)$ o $\left.\left(\mathrm{Y}_{\mathrm{n}}, \mathrm{X}_{\mathrm{n}}, \mathrm{Z}_{\mathrm{n}}\right)\right)$ estarán siempre asociados a los ejes estructurales $\mathrm{N}$ $\mathrm{S}$, E-W y V, respectivamente. Para obtener las respuestas de referencia cuando se aplican las dos componentes horizontales, normales y principales, se consideran los siguientes cuatro casos de carga:

Caso 1: Los modelos se excitan simultáneamente por las componentes normales, la primera componente horizontal actúa en la dirección N-S y la segunda componente horizontal en la dirección E-W. Este caso se denota como $\left(\mathrm{X}_{\mathrm{n}}, \mathrm{Y}_{\mathrm{n}}, 0\right)$.

Caso 2: Igual al Caso 1, pero las componentes se intercambian $\left(Y_{n}, X_{n}, 0\right)$, es decir, la segunda componente horizontal actúa ahora en la dirección N-S y la primera componente horizontal en la dirección E-W.

Caso 3: Igual al Caso 1, pero los modelos se excitan por las componentes principales $\left(\mathrm{X}_{\mathrm{p}}, \mathrm{Y}_{\mathrm{p}}, 0\right)$.

Caso 4: Igual al Caso 2, pero los modelos se excitan por las componentes principales $\left(\mathrm{Y}_{\mathrm{p}}, \mathrm{X}_{\mathrm{p}}, 0\right)$.

Para obtener las respuestas de referencia cuando los modelos se excitan por las tres componentes normales y principales, se requieren los siguientes cuatro casos de carga adicionales: 
Caso 5: Los modelos se excitan por las tres componentes normales, la primera componente horizontal actúa en la dirección N-S, la segunda componente horizontal en la dirección E-W y la tercera componente en la dirección vertical. Este caso se denota como $\left(\mathrm{X}_{\mathrm{n}}, \mathrm{Y}_{\mathrm{n}}, \mathrm{Z}_{\mathrm{n}}\right)$.

Caso 6: Igual al Caso 5, pero las componentes horizontales se intercambian $\left(\mathrm{Y}_{\mathrm{n}}, \mathrm{X}_{\mathrm{n}}, \mathrm{Z}_{\mathrm{n}}\right)$, es decir, la segunda componente horizontal actúa en la dirección N-S y la primera componente horizontal en la dirección E-W.

Caso 7: Igual al Caso 5, pero se aplican las componentes principales $\left(\mathrm{X}_{\mathrm{p}}, \mathrm{Y}_{\mathrm{p}}, \mathrm{Z}_{\mathrm{p}}\right)$.

Caso 8: Igual al Caso 6, pero se aplican las componentes principales $\left(\mathrm{Y}_{\mathrm{p}}, \mathrm{X}_{\mathrm{p}}, \mathrm{Z}_{\mathrm{p}}\right)$.

Para cualquier parámetro de respuesta colineal (carga axial) o no colineal (cortantes de entrepiso, cortante basal), la respuesta de referencia cuando los modelos se excitan por las dos componentes horizontales normales, denotada en lo sucesivo como $\mathrm{R}_{\mathrm{n} 2}$, será la máxima respuesta de los Casos 1 y 2 , mientras que para las componentes horizontales principales, la respuesta de referencia, $\mathrm{R}_{\mathrm{p} 2}$, será la máxima respuesta de los Casos 3 y 4. Similarmente, cuando las estructuras se excitan por las tres componentes, la respuesta de referencia para componentes normales, denotada en lo sucesivo como $\mathrm{R}_{\mathrm{n} 3}$, será la máxima respuesta de los Casos 5 y 6. La correspondiente respuesta de referencia para componentes principales, $\mathrm{R}_{\mathrm{p} 3}$, será la máxima respuesta de los Casos 7 y 8 .

Para evaluar la precisión de las reglas de combinación, es necesario obtener las respuestas individuales en cada dirección ortogonal. Es importante mencionar que los códigos de diseño sísmico especifican un solo espectro de diseño para análisis multi-componente, es decir, se aplica el mismo espectro en cada dirección horizontal de la estructura. En este trabajo se evalúa la precisión de las reglas de combinación aplicando cada una de las componentes (normales y principales) de los registros sísmicos en cada dirección ortogonal considerando los siguientes cuatro casos de carga:

Caso 9: De acuerdo a las reglas del 30\% y SRSS, los modelos se analizan considerando cada componente actuando de manera individual, para lo que se tiene los siguientes 3 sub-casos para componentes normales: a) $\left.\left(X_{n}, 0,0\right), b\right)\left(0, Y_{n}, 0\right)$ y c) $\left(0,0, Z_{n}\right)$.

Caso 10: Igual al Caso 9, pero se intercambian las componentes horizontales: a) $\left.\left(Y_{n}, 0,0\right), b\right)\left(0, X_{n}, 0\right)$ y c) $\left(0,0, Z_{\mathrm{n}}\right)$.

Caso 11: Igual al Caso 9, pero se aplican las componentes principales: a) $\left.\left(X_{p}, 0,0\right), b\right)\left(0, Y_{p}, 0\right)$ y c) $\left(0,0, Z_{\mathrm{p}}\right)$.

Caso 12: Igual al Caso 10, pero se aplican las componentes principales: a) $\left.\left(Y_{p}, 0,0\right), b\right)\left(0, X_{p}, 0\right)$ y c) $\left(0,0, Z_{\mathrm{p}}\right)$

Entonces, para dos modelos, veinte registros sísmicos, doce casos de carga y considerando comportamiento elástico e inelástico, se requieren un total de 960 análisis de SVGL. Para la estimación del ángulo de incidencia crítico, las componentes horizontales se rotan con incrementos de $5^{\circ}$. En este caso, se requerirán un total de 1520 análisis. Por lo tanto, en esta investigación se realizaron aproximadamente 2500 análisis sísmicos de SVGL.

\section{PRECISIÓN DE LAS REGLAS DE COMBINACIÓN}

La precisión de las reglas de combinación en la estimación de la respuesta combinada se discute a continuación. Se consideraron parámetros de respuesta locales y globales. Se muestran los resultados para dos y tres componentes. 


\section{Regla del 30\%, comportamiento elástico}

Considerando componentes normales, la respuesta combinada se puede calcular de tres maneras. Por ejemplo para el Caso de Carga 9 dichas maneras son: a) ${ }^{9} X_{n}+0.30{ }^{9} Y_{n}+0.30{ }^{9} Z_{n}$, b) $0.30{ }^{9} X_{n}+{ }^{9} Y_{n}+0.30{ }^{9} Z_{n}$ y c) $0.30{ }^{9} X_{n}+0.30{ }^{9} Y_{n}+{ }^{9} Z_{n}$, donde ${ }^{9} X_{n},{ }^{9} Y_{n} y{ }^{9} Z_{n}$ se definen como las respuestas producidas por los casos de carga 9a, 9b y 9c, respectivamente. La mayor de las tres respuestas combinadas se normaliza con respecto a la respuesta de referencia $\left(\mathrm{R}_{\mathrm{n} 3}\right)$ definida anteriormente. Los valores normalizados se denotarán como ${ }^{9} R_{n 3330}$. Siguiendo exactamente el mismo procedimiento para el Caso 10, se calculan los valores de ${ }^{10} R_{n 3,30}$. La combinación de ambos casos $\left({ }^{9} R_{n 3,30}\right.$, ${ }^{10} \mathrm{R}_{\mathrm{n} 3,30}$ ) y 20 terremotos resultarán en 40 valores que serán representados por la variable aleatoria $\mathrm{R}_{\mathrm{n} 3,30}$. Similarmente, considerando los Casos de Carga 11 y 12 para las componentes principales, se calcula la variable $R_{\mathrm{p} 330}$. Para el caso en que se consideren solo las dos componentes horizontales, se sigue un procedimiento similar al descrito anteriormente, lo cual resulta en las variables aleatorias $R_{\mathrm{n} 2,30}$ y $R_{\mathrm{p} 2,30}$. En la Figuras $2 \mathrm{a}$ y $2 \mathrm{~b}$ se muestran los valores calculados de $R_{\mathrm{n} 2,30}$ y $R_{\mathrm{n} 3,30}$, respectivamente, para carga axial en las columnas del Modelo 1. Se observa que ambos parámetros varían significativamente de un sismo a otro y de elemento a otro sin mostrar tendencia alguna. En la mayoría de los casos la regla del 30\% subestima la respuesta combinada. Los resultados para los parámetros $R_{p 2,30} R_{p 3,30}$ (Figuras 2 c y $2 d$ ) correspondientes a las componentes principales siguen una tendencia similar.

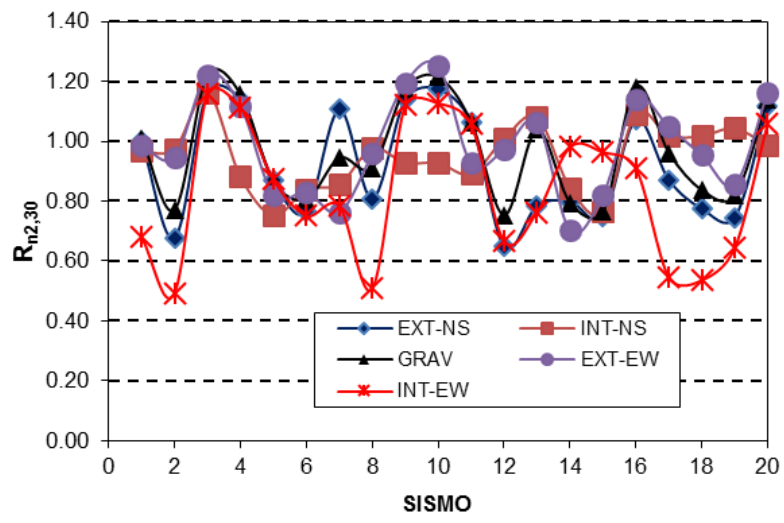

a) Componentes normales, dos componentes

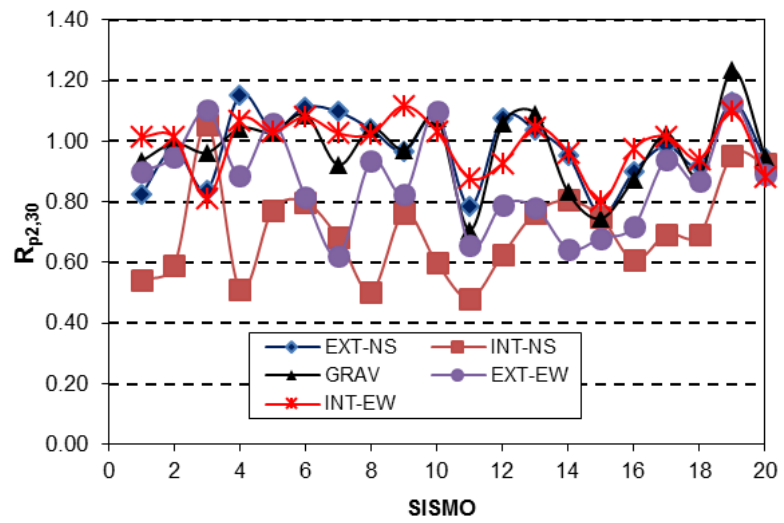

c) Componentes principales, dos componentes

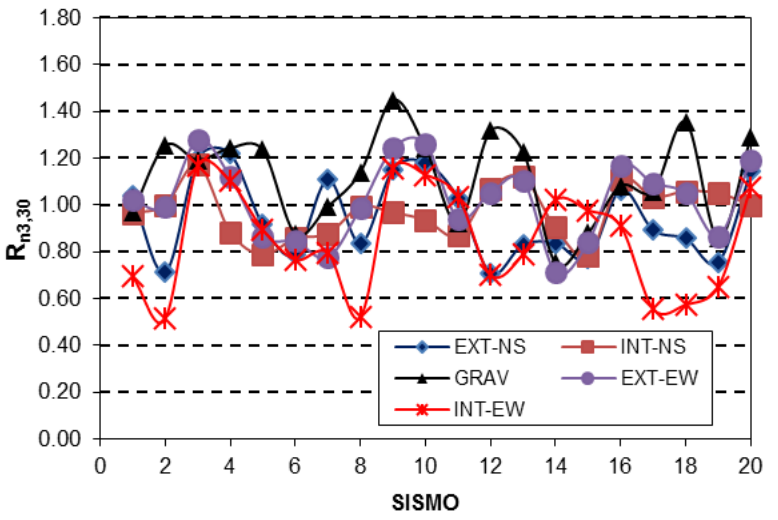

b) Componentes normales, tres componentes

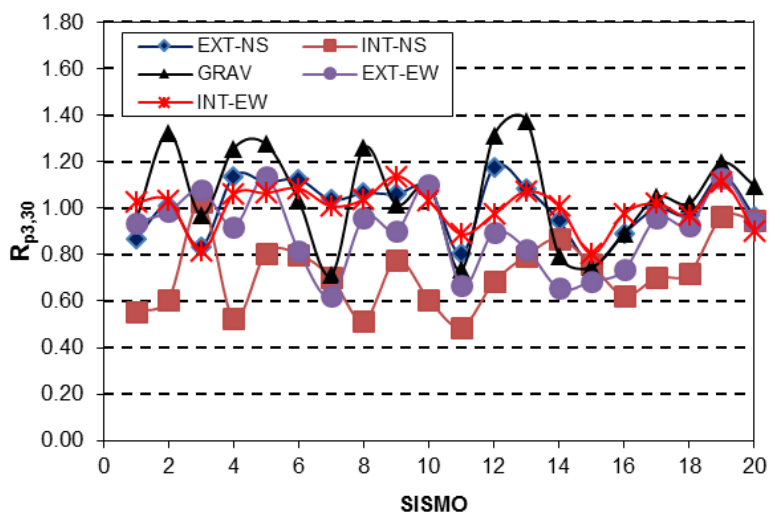

d) Componentes principales, tres componentes

Figura 2. Precisión de la regla del 30\%, carga axial, comportamiento elástico, Modelo 1 
La precisión de la regla del $30 \%$ se discute ahora para cortantes de entrepiso. Los parámetros $\mathrm{R}_{\mathrm{n} 2,30 \text {, }}$ $\mathbf{R}_{\mathrm{p} 2,30}, \mathbf{R}_{\mathrm{n} 3,30}$ y $\mathbf{R}_{\mathrm{p} 3,30}$ se calculan de la misma manera que para carga axial, la única diferencia es que ahora representan cortantes de entrepiso. Las gráficas correspondientes se muestran en la Figura 3. La principal observación que puede hacerse es que la respuesta combinada se sobreestima en la gran mayoría de los casos. Las estadísticas para carga axial y cortante de entrepiso se dan en las Tablas 3 y 4, para dos y tres componentes, respectivamente. Los resultados en las tablas indican que para carga axial y dos componentes, la regla del $30 \%$, en promedio, subestima la carga axial alrededor del $10 \%$ y que la incertidumbre (COV) asociada a dicha subestimación es alrededor del $20 \%$. Por otra parte, el cortante se sobreestima, en promedio, en un $10 \%$ para ambos modelos. La incertidumbre en la estimación es mucho mayor para carga axial que para cortante. Además, se puede observar que la regla del $30 \%$ es ligeramente más precisa en estimar la carga axial cuando se consideran las tres componentes de los terremotos que solo cuando se consideran las componentes horizontales. En el caso de los cortantes de entrepiso, no se observan diferencias significativas entre dos y tres componentes.

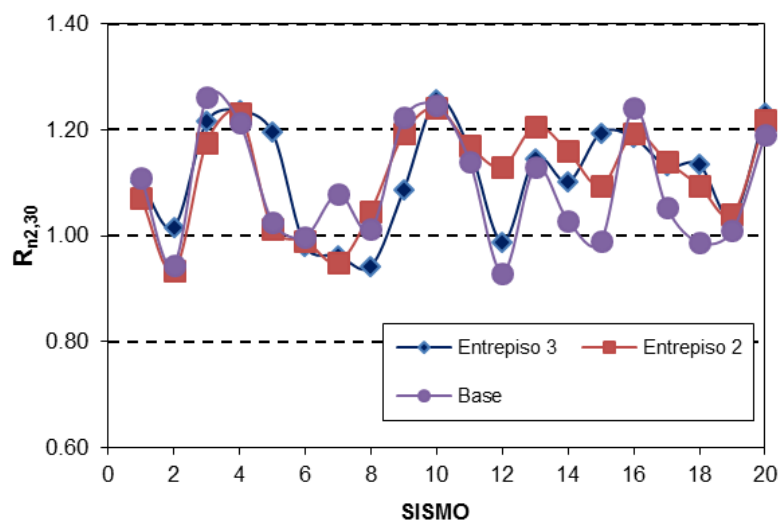

a) Componentes normales, dos componentes

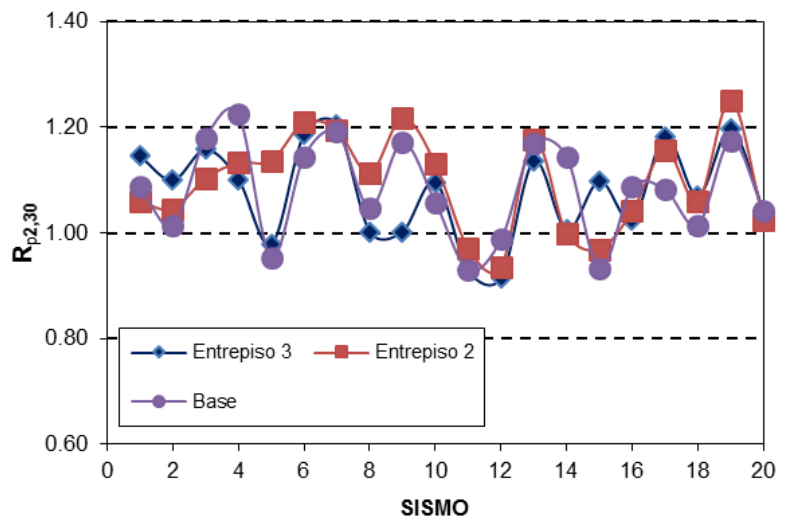

c) Componentes principales, dos componentes

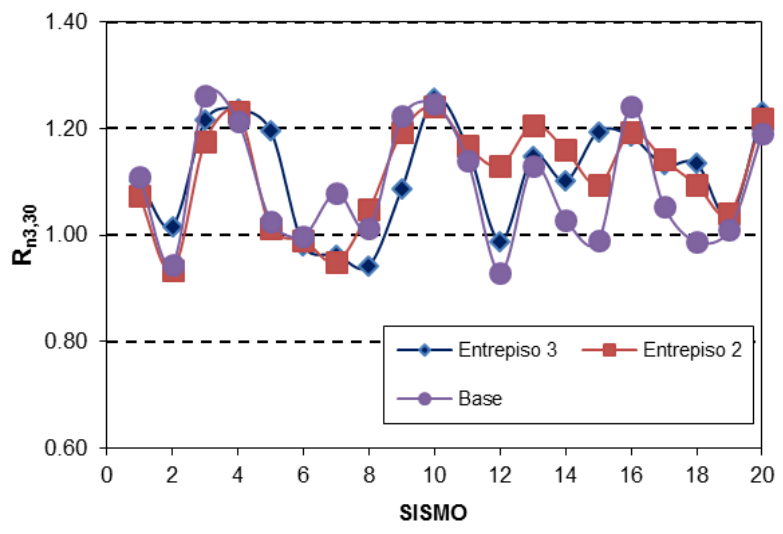

b) Componentes normales, tres componentes

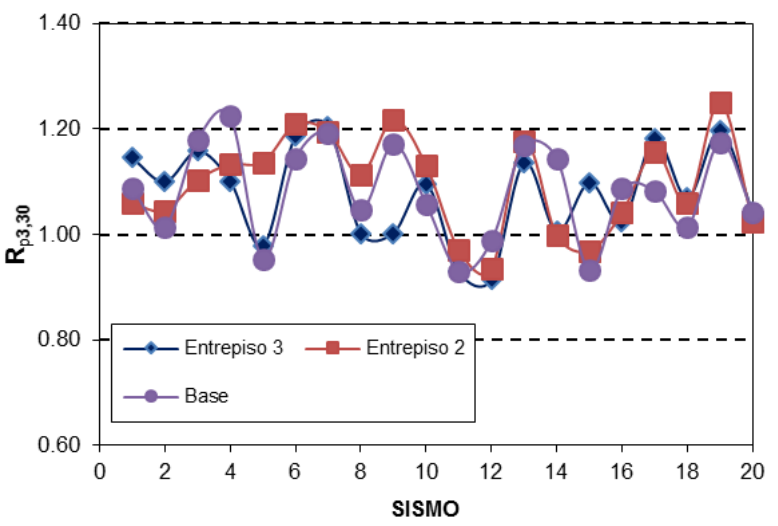

d) Componentes principales, tres componentes

Figura 3. Precisión de la regla del 30\%, cortantes de entrepiso, comportamiento elástico, Modelo 1 
Tabla 3. Estadísticas para $\mathrm{R}_{\mathrm{n} 2,30}, \mathrm{R}_{\mathrm{p} 2,30}, \mathrm{R}_{\mathrm{n} 2, \mathrm{SRSS}}$ y $\mathrm{R}_{\mathrm{p} 2, \mathrm{SRSS}}$, carga axial y cortantes de entrepiso, comportamiento elástico

\begin{tabular}{|c|c|c|c|c|c|c|c|c|c|c|c|}
\hline \multirow{3}{*}{$\begin{array}{l}\text { Modelo } \\
\text { (1) }\end{array}$} & \multirow{3}{*}{\multicolumn{2}{|c|}{$\begin{array}{l}\text { Localización } \\
\text { (2) }\end{array}$}} & \multicolumn{4}{|c|}{ Regla del $30 \%$} & \multicolumn{4}{|c|}{ Regla SRSS } & \multirow{3}{*}{$\begin{array}{l}\text { Tamaño de } \\
\text { la muestra } \\
\text { (11) }\end{array}$} \\
\hline & & & \multicolumn{2}{|c|}{$\mathrm{R}_{\mathrm{n} 2,30}$} & \multicolumn{2}{|c|}{$\mathrm{R}_{\mathrm{p} 2,30}$} & \multicolumn{2}{|c|}{$\mathrm{R}_{\mathrm{n} 2, \mathrm{SRSS}}$} & \multicolumn{2}{|c|}{$\mathrm{R}_{\mathrm{P} 2, \mathrm{SRSS}}$} & \\
\hline & & & $\begin{array}{c}\mu \\
(3)\end{array}$ & $\begin{array}{c}\mathrm{COV} \\
(4)\end{array}$ & $\begin{array}{c}\mu \\
(5)\end{array}$ & $\begin{array}{c}\mathrm{COV} \\
(6)\end{array}$ & $\begin{array}{c}\mu \\
(7)\end{array}$ & $\begin{array}{c}\mathrm{COV} \\
(8)\end{array}$ & $\begin{array}{c}\mu \\
(9)\end{array}$ & $\begin{array}{l}\text { COV } \\
(10)\end{array}$ & \\
\hline \multirow{10}{*}{1} & \multirow{6}{*}{$\begin{array}{l}\text { Carga } \\
\text { axial }\end{array}$} & EXT-NS & 0.89 & 0.18 & 0.91 & 0.16 & 0.87 & 0.18 & 0.90 & 0.15 & 40 \\
\hline & & INT-NS & 0.87 & 0.20 & 0.84 & 0.22 & 0.90 & 0.18 & 0.83 & 0.20 & 40 \\
\hline & & GRAV & 1.00 & 0.16 & 1.02 & 0.14 & 1.01 & 0.17 & 1.00 & 0.14 & 40 \\
\hline & & EXT-EW & 0.92 & 0.16 & 0.93 & 0.17 & 0.85 & 0.17 & 0.93 & 0.17 & 40 \\
\hline & & INT-EW & 0.89 & 0.23 & 0.86 & 0.22 & 0.89 & 0.21 & 0.85 & 0.20 & 40 \\
\hline & & Todos & 0.91 & 0.19 & 0.91 & 0.19 & 0.90 & 0.19 & 0.90 & 0.18 & 200 \\
\hline & \multirow{4}{*}{ Cortante } & ENT 3 & 1.12 & 0.07 & 1.08 & 0.08 & 1.13 & 0.09 & 1.10 & 0.08 & 40 \\
\hline & & ENT 2 & 1.12 & 0.08 & 1.10 & 0.08 & 1.14 & 0.10 & 1.12 & 0.08 & 40 \\
\hline & & BASE & 1.10 & 0.09 & 1.10 & 0.07 & 1.12 & 0.10 & 1.11 & 0.08 & 40 \\
\hline & & Todos & 1.12 & 0.08 & 1.09 & 0.08 & 1.13 & 0.10 & 1.11 & 0.08 & 120 \\
\hline \multirow{16}{*}{2} & \multirow{6}{*}{$\begin{array}{c}\text { Carga } \\
\text { axial }\end{array}$} & EXT-NS & 0.91 & 0.15 & 0.88 & 0.22 & 0.89 & 0.13 & 0.87 & 0.22 & 40 \\
\hline & & INT-NS & 0.87 & 0.14 & 0.84 & 0.09 & 0.86 & 0.14 & 0.88 & 0.09 & 40 \\
\hline & & GRAV & 1.01 & 0.14 & 1.00 & 0.10 & 0.99 & 0.15 & 1.02 & 0.10 & 40 \\
\hline & & EXT-EW & 0.82 & 0.14 & 0.87 & 0.23 & 0.85 & 0.14 & 0.86 & 0.24 & 40 \\
\hline & & INT-EW & 0.88 & 0.14 & 0.90 & 0.10 & 0.88 & 0.14 & 0.91 & 0.10 & 40 \\
\hline & & Todos & 0.90 & 0.14 & 0.90 & 0.16 & 0.89 & 0.14 & 0.91 & 0.16 & 200 \\
\hline & \multirow{10}{*}{ Cortante } & ENT 9 & 1.12 & 0.06 & 1.08 & 0.07 & 1.12 & 0.08 & 1.09 & 0.05 & 40 \\
\hline & & ENT 8 & 1.12 & 0.05 & 1.09 & 0.07 & 1.13 & 0.07 & 1.09 & 0.06 & 40 \\
\hline & & ENT 7 & 1.13 & 0.05 & 1.09 & 0.07 & 1.13 & 0.07 & 1.09 & 0.06 & 40 \\
\hline & & ENT 6 & 1.12 & 0.06 & 1.09 & 0.07 & 1.13 & 0.07 & 1.09 & 0.06 & 40 \\
\hline & & ENT 5 & 1.12 & 0.07 & 1.10 & 0.06 & 1.13 & 0.08 & 1.10 & 0.05 & 40 \\
\hline & & ENT 4 & 1.11 & 0.07 & 1.10 & 0.06 & 1.13 & 0.08 & 1.10 & 0.07 & 40 \\
\hline & & ENT 3 & 1.12 & 0.07 & 1.11 & 0.06 & 1.15 & 0.09 & 1.10 & 0.07 & 40 \\
\hline & & ENT 2 & 1.13 & 0.07 & 1.12 & 0.05 & 1.15 & 0.09 & 1.11 & 0.07 & 40 \\
\hline & & BASE & 1.14 & 0.08 & 1.13 & 0.05 & 1.16 & 0.09 & 1.13 & 0.06 & 40 \\
\hline & & Todos & 1.12 & 0.06 & 1.10 & 0.06 & 1.14 & 0.08 & 1.10 & 0.06 & 360 \\
\hline
\end{tabular}


Tabla 4. Estadísticas para $\mathrm{R}_{\mathrm{n} 3,30}, \mathrm{R}_{\mathrm{p} 3,30}, \mathrm{R}_{\mathrm{n} 3, \mathrm{SRSS}}$ y $\mathrm{R}_{\mathrm{p} 3, \mathrm{SRSS}}$, carga axial y cortantes de entrepiso, comportamiento elástico

\begin{tabular}{|c|c|c|c|c|c|c|c|c|c|c|c|}
\hline \multirow{3}{*}{$\begin{array}{l}\text { Modelo } \\
\text { (1) }\end{array}$} & \multirow{3}{*}{\multicolumn{2}{|c|}{$\begin{array}{l}\text { Localización } \\
\text { (2) }\end{array}$}} & \multicolumn{4}{|c|}{ Regla del $30 \%$} & \multicolumn{4}{|c|}{ Regla SRSS } & \multirow{3}{*}{$\begin{array}{c}\text { Tamaño de } \\
\text { la muestra } \\
\quad(11)\end{array}$} \\
\hline & & & \multicolumn{2}{|c|}{$\mathrm{R}_{\mathrm{n} 3,30}$} & \multicolumn{2}{|c|}{$\mathrm{R}_{\mathrm{p} 3,30}$} & \multicolumn{2}{|c|}{$\mathrm{R}_{\mathrm{n} 3, \mathrm{SRSS}}$} & \multicolumn{2}{|c|}{$\mathrm{R}_{\mathrm{P} 3, \mathrm{SRSS}}$} & \\
\hline & & & $\begin{array}{c}\mu \\
(3)\end{array}$ & $\begin{array}{l}\mathrm{COV} \\
(4)\end{array}$ & $\begin{array}{c}\mu \\
(5)\end{array}$ & $\begin{array}{c}\mathrm{COV} \\
(6)\end{array}$ & $\begin{array}{c}\mu \\
(7)\end{array}$ & $\begin{array}{c}\mathrm{COV} \\
(8)\end{array}$ & $\begin{array}{c}\mu \\
(9)\end{array}$ & $\begin{array}{l}\mathrm{COV} \\
(10)\end{array}$ & \\
\hline \multirow{10}{*}{1} & \multirow{6}{*}{$\begin{array}{l}\text { Carga } \\
\text { axial }\end{array}$} & EXT-NS & 0.94 & 0.17 & 0.93 & 0.15 & 0.94 & 0.17 & 0.92 & 0.15 & 40 \\
\hline & & INT-NS & 0.90 & 0.19 & 0.86 & 0.22 & 0.90 & 0.18 & 0.86 & 0.20 & 40 \\
\hline & & GRAV & 1.07 & 0.17 & 1.05 & 0.19 & 1.11 & 0.17 & 1.02 & 0.19 & 40 \\
\hline & & EXT-EW & 0.95 & 0.16 & 0.96 & 0.17 & 0.98 & 0.17 & 0.93 & 0.17 & 40 \\
\hline & & INT-EW & 0.90 & 0.22 & 0.87 & 0.21 & 0.87 & 0.20 & 0.88 & 0.19 & 40 \\
\hline & & Todos & 0.95 & 0.20 & 0.94 & 0.20 & 0.96 & 0.20 & 0.92 & 0.20 & 200 \\
\hline & \multirow{4}{*}{ Cortante } & ENT 3 & 1.12 & 0.08 & 1.08 & 0.08 & 1.13 & 0.09 & 1.10 & 0.08 & 40 \\
\hline & & ENT 2 & 1.12 & 0.08 & 1.10 & 0.08 & 1.14 & 0.10 & 1.12 & 0.08 & 40 \\
\hline & & BASE & 1.10 & 0.09 & 1.10 & 0.07 & 1.12 & 0.10 & 1.11 & 0.08 & 40 \\
\hline & & Todos & 1.11 & 0.08 & 1.09 & 0.08 & 1.13 & 0.10 & 1.11 & 0.08 & 120 \\
\hline \multirow{16}{*}{2} & \multirow{6}{*}{$\begin{array}{c}\text { Carga } \\
\text { axial }\end{array}$} & EXT-NS & 0.92 & 0.15 & 0.90 & 0.21 & 0.94 & 0.14 & 0.88 & 0.22 & 40 \\
\hline & & INT-NS & 0.98 & 0.14 & 0.96 & 0.09 & 0.98 & 0.14 & 0.97 & 0.09 & 40 \\
\hline & & GRAV & 1.06 & 0.18 & 1.04 & 0.15 & 1.05 & 0.18 & 1.01 & 0.14 & 40 \\
\hline & & EXT-EW & 0.88 & 0.15 & 0.89 & 0.22 & 0.86 & 0.14 & 0.89 & 0.23 & 40 \\
\hline & & INT-EW & 0.94 & 0.14 & 0.98 & 0.10 & 0.92 & 0.15 & 0.93 & 0.10 & 40 \\
\hline & & Todos & 0.96 & 0.16 & 0.95 & 0.17 & 0.95 & 0.16 & 0.94 & 0.17 & 200 \\
\hline & \multirow{10}{*}{ Cortante } & ENT 9 & 1.12 & 0.07 & 1.08 & 0.08 & 1.12 & 0.08 & 1.09 & 0.05 & 40 \\
\hline & & ENT 8 & 1.12 & 0.06 & 1.09 & 0.08 & 1.13 & 0.07 & 1.09 & 0.06 & 40 \\
\hline & & ENT 7 & 1.13 & 0.06 & 1.09 & 0.08 & 1.13 & 0.07 & 1.09 & 0.06 & 40 \\
\hline & & ENT 6 & 1.12 & 0.06 & 1.09 & 0.07 & 1.13 & 0.07 & 1.09 & 0.06 & 40 \\
\hline & & ENT 5 & 1.12 & 0.08 & 1.10 & 0.06 & 1.13 & 0.08 & 1.10 & 0.05 & 40 \\
\hline & & ENT 4 & 1.11 & 0.08 & 1.10 & 0.07 & 1.13 & 0.08 & 1.10 & 0.07 & 40 \\
\hline & & ENT 3 & 1.12 & 0.08 & 1.11 & 0.07 & 1.15 & 0.09 & 1.10 & 0.07 & 40 \\
\hline & & ENT 2 & 1.13 & 0.08 & 1.12 & 0.06 & 1.15 & 0.09 & 1.11 & 0.07 & 40 \\
\hline & & BASE & 1.14 & 0.09 & 1.13 & 0.05 & 1.16 & 0.09 & 1.13 & 0.06 & 40 \\
\hline & & Todos & 1.12 & 0.06 & 1.10 & 0.06 & 1.14 & 0.08 & 1.10 & 0.06 & 360 \\
\hline
\end{tabular}

\section{Regla SRSS, comportamiento elástico}

La precisión de la regla SRSS en la estimación de la respuesta combinada se evalúa siguiendo el mismo procedimiento que para la regla del $30 \%$. Las variables aleatorias correspondientes que definen dicha precisión se denotan como $R_{\mathrm{n} 2 \text {,SRSS }}$ $\mathrm{R}_{\mathrm{n} 3 \text {,SRSS }}$ (para componentes normales), y $\mathrm{R}_{\mathrm{p} 2 \text {,SRss }}$ y $\mathrm{R}_{\mathrm{p} 3 \text {,SRSS }}$ (para componentes principales). Los valores calculados de estos parámetros se presentan en la Figura 4 para carga axial en las columnas del Modelo 2. Las principales observaciones hechas para la regla del 30\% también aplican para la regla SRSS: los valores de los parámetros varían de un elemento a otro y de un sismo a otro sin mostrar tendencia alguna. Se observan valores de subestimación cercanos al $60 \%$ en 
varios casos, incluso para componentes no correlacionadas (Figs. 4c y 4d). Las estadísticas correspondientes se muestran en las columnas (7) a la (10) de las Tablas 3 y 4. Los resultados indican que al igual que en el caso de la regla del $30 \%$, en promedio, la regla SRSS subestima la carga axial. Los niveles de subestimación y de incertidumbre son esencialmente iguales para ambas reglas de combinación. No se observan diferencias significativas entre componentes normales y principales. La respuesta en términos de cortante también se sobreestima en este caso. Al igual que para la regla del 30\%, la regla SRSS estima mejor la respuesta combinada en términos de carga axial cuando se consideran las tres componentes de los terremotos.

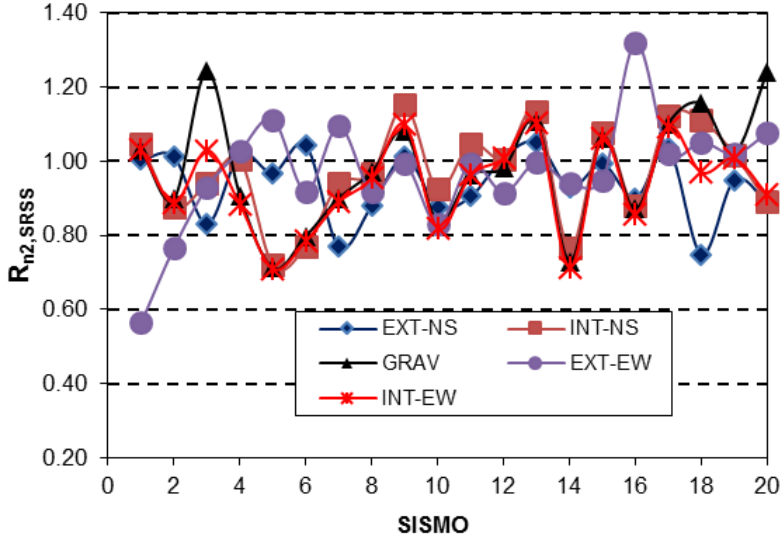

a) Componentes normales, dos componentes

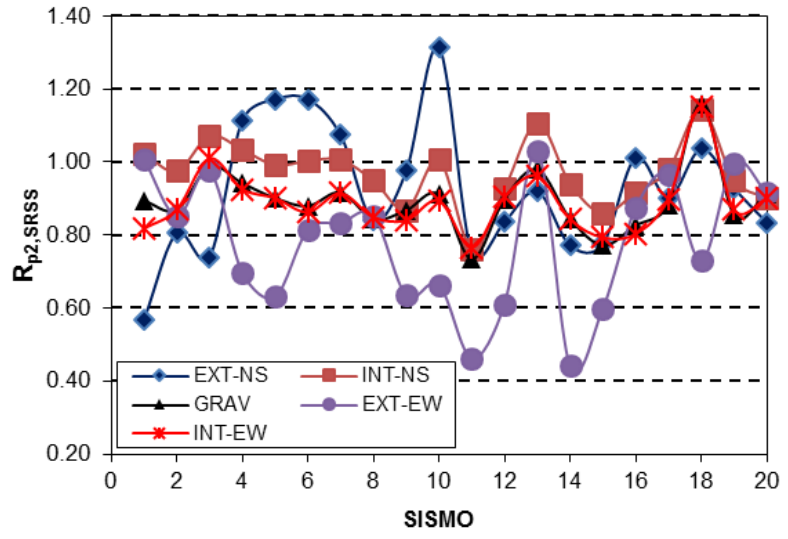

c) Componentes principales, dos componentes

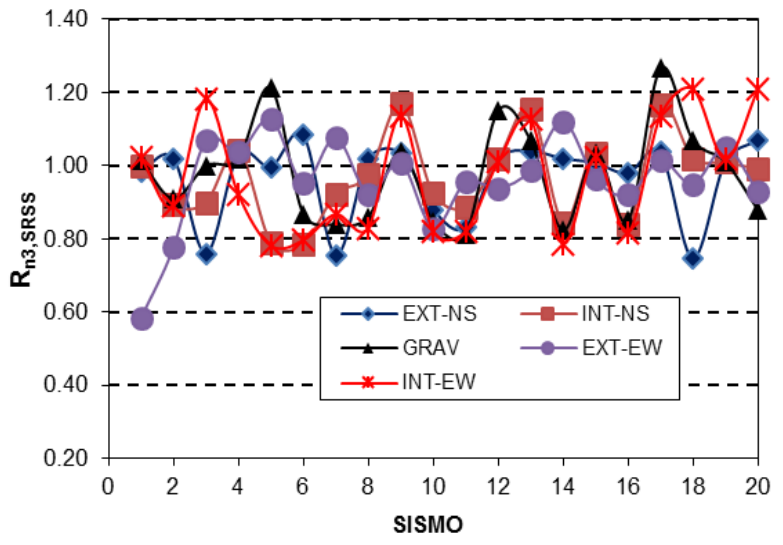

b) Componentes normales, tres componentes

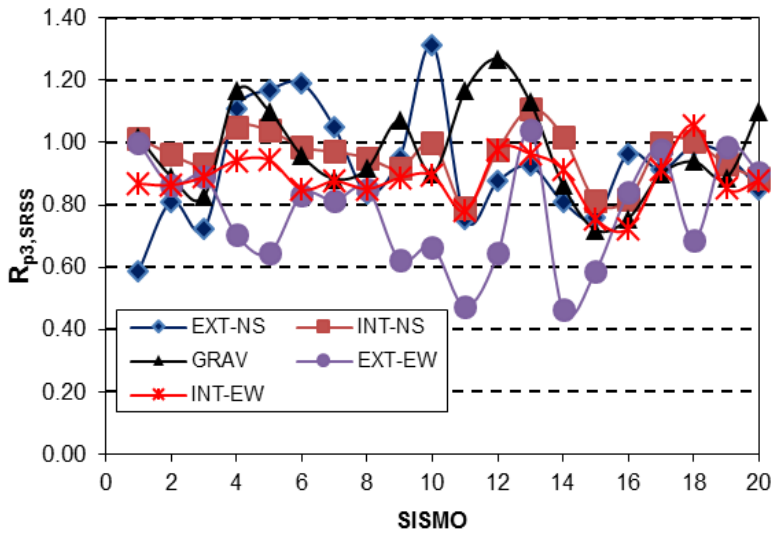

d) Componentes principales, tres componentes

Figura 4. Precisión de la regla SRSS, carga axial, comportamiento elástico, Modelo 2

\section{Reglas del 30\% y SRSS, comportamiento inelástico}

La precisión de las reglas también se estudia para el caso en que los modelos incursionan en el rango inelástico. Las gráficas correspondientes se presentan en la Figura 5. Se observa un comportamiento muy similar al caso elástico: los valores de los parámetros varían de un sismo a otro y de un elemento a otro sin mostrar tendencias. En las Tablas 5 y 6 se presentan las estadísticas correspondientes para ambos modelos y reglas de combinación, para dos y tres componentes, respectivamente. Como se puede ver en las tablas, ambas reglas, en promedio, subestiman la carga axial alrededor de $15 \%$. La incertidumbre en la estimación es mayor a $30 \%$. El cortante se sobreestima alrededor de $10 \%$ y la incertidumbre es mucho menor que para carga axial. No se observan diferencias significativas entre ambas reglas de combinación 
ni entre componentes normales y principales. En comparación al caso elástico, se puede observar que los niveles de subestimación y de incertidumbre aumentan cuando se considera comportamiento inelástico. En este caso las reglas también son ligeramente más precisas para tres que para dos componentes.

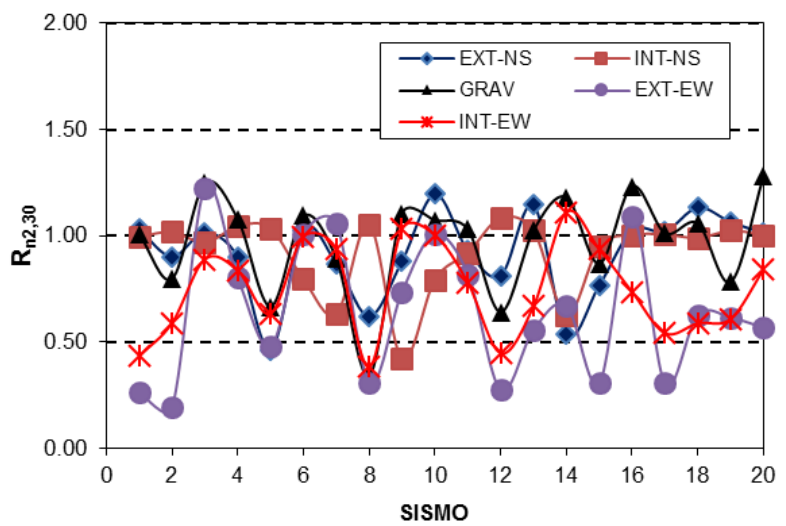

a) Regla del $30 \%$, dos componentes

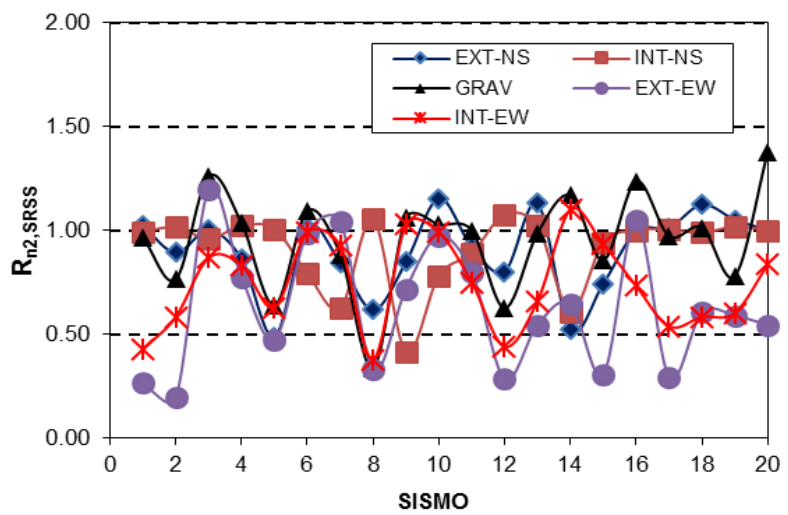

c) Regla SRSS, dos componentes

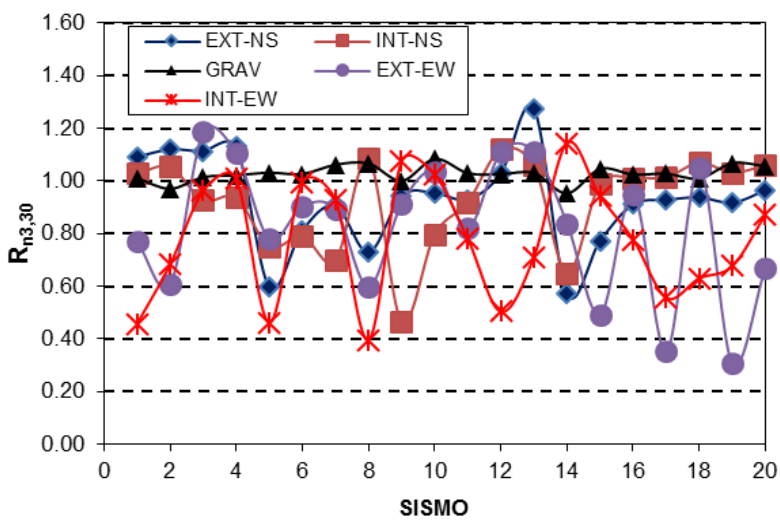

b) Regla del $30 \%$, tres componentes

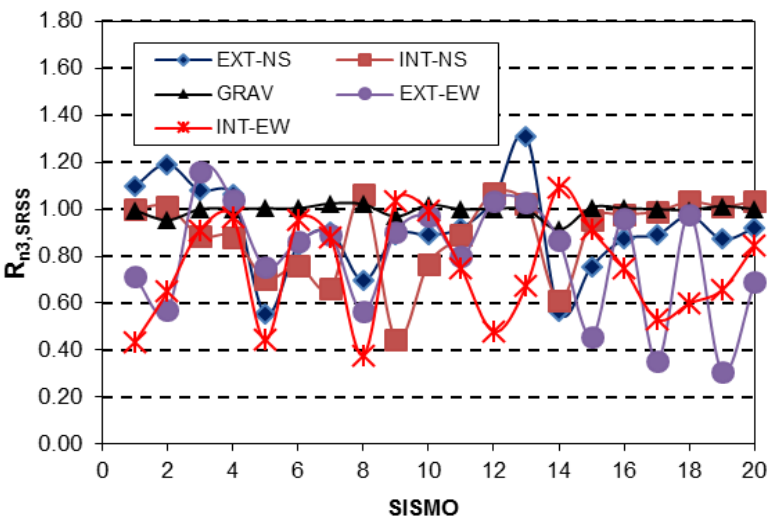

d) Regla SRSS, tres componentes

Figura 5. Precisión de las reglas de combinación, carga axial, componentes normales, comportamiento inelástico, Modelo 1

Tabla 5. Estadísticas para $\mathrm{R}_{\mathrm{n} 2,30}, \mathrm{R}_{\mathrm{p} 2,30}, \mathrm{R}_{\mathrm{n} 2, \mathrm{SRSS}}$ y $\mathrm{R}_{\mathrm{p} 2, \mathrm{SRSS}}$, carga axial y cortantes de entrepiso, comportamiento inelástico

\begin{tabular}{|c|c|c|c|c|c|c|c|c|c|c|c|}
\hline \multirow{3}{*}{$\begin{array}{l}\text { Modelo } \\
\text { (1) }\end{array}$} & \multirow{3}{*}{\multicolumn{2}{|c|}{$\begin{array}{l}\text { Localización } \\
\text { (2) }\end{array}$}} & \multicolumn{4}{|c|}{ Regla del $30 \%$} & \multicolumn{4}{|c|}{ Regla SRSS } & \multirow{3}{*}{$\begin{array}{l}\text { Tamaño de } \\
\text { la muestra } \\
\text { (11) }\end{array}$} \\
\hline & & & \multicolumn{2}{|c|}{$\mathrm{R}_{\mathrm{n} 2,30}$} & \multicolumn{2}{|c|}{$\mathrm{R}_{\mathrm{p} 2,30}$} & \multicolumn{2}{|c|}{$\mathrm{R}_{\mathrm{n} 2 \text {,SRSS }}$} & \multicolumn{2}{|c|}{$\mathrm{R}_{\mathrm{p} 2 \text {,SRSS }}$} & \\
\hline & & & $\begin{array}{c}\mu \\
(3)\end{array}$ & $\begin{array}{l}\text { COV } \\
(4)\end{array}$ & $\begin{array}{c}\mu \\
(5)\end{array}$ & $\begin{array}{c}\mathrm{COV} \\
(6)\end{array}$ & $\begin{array}{c}\mu \\
(7)\end{array}$ & $\begin{array}{c}\mathrm{COV} \\
(8)\end{array}$ & $\begin{array}{c}\mu \\
(9)\end{array}$ & $\begin{array}{l}\mathrm{COV} \\
(10)\end{array}$ & \\
\hline \multirow{6}{*}{1} & \multirow{6}{*}{$\begin{array}{c}\text { Carga } \\
\text { axial }\end{array}$} & EXT-NS & 0.90 & 0.40 & 0.91 & 0.37 & 0.88 & 0.41 & 0.89 & 0.37 & 40 \\
\hline & & INT-NS & 0.87 & 0.25 & 0.88 & 0.19 & 0.86 & 0.24 & 0.87 & 0.20 & 40 \\
\hline & & GRAV & 0.95 & 0.23 & 0.90 & 0.19 & 0.94 & 0.23 & 0.89 & 0.18 & 40 \\
\hline & & EXT-EW & 0.79 & 0.46 & 0.75 & 0.51 & 0.77 & 0.47 & 0.74 & 0.59 & 40 \\
\hline & & INT-EW & 0.85 & 0.27 & 0.91 & 0.23 & 0.84 & 0.27 & 0.90 & 0.23 & 40 \\
\hline & & Todos & 0.87 & 0.33 & 0.87 & 0.31 & 0.86 & 0.33 & 0.86 & 0.34 & 200 \\
\hline
\end{tabular}


Tabla 5. Estadísticas para $\mathrm{R}_{\mathrm{n} 2,30}, \mathrm{R}_{\mathrm{p} 2,30}, \mathrm{R}_{\mathrm{n} 2, \mathrm{SRSS}}$ y $\mathrm{R}_{\mathrm{p} 2, \mathrm{SRSS}}$, carga axial y cortantes de entrepiso, comportamiento inelástico (continuación)

\begin{tabular}{|c|c|c|c|c|c|c|c|c|c|c|c|}
\hline \multirow{3}{*}{$\begin{array}{l}\text { Modelo } \\
\text { (1) }\end{array}$} & \multirow{3}{*}{\multicolumn{2}{|c|}{$\begin{array}{l}\text { Localización } \\
\text { (2) }\end{array}$}} & \multicolumn{4}{|c|}{ Regla del $30 \%$} & \multicolumn{4}{|c|}{ Regla SRSS } & \multirow{3}{*}{$\begin{array}{l}\text { Tamaño de } \\
\text { la muestra } \\
\text { (11) }\end{array}$} \\
\hline & & & \multicolumn{2}{|c|}{$\mathrm{R}_{\mathrm{n} 2,30}$} & \multicolumn{2}{|c|}{$\mathrm{R}_{\mathrm{p} 2,30}$} & \multicolumn{2}{|c|}{$\mathrm{R}_{\mathrm{n} 2 \text {,SRSS }}$} & \multicolumn{2}{|c|}{$\mathrm{R}_{\mathrm{p} 2 \text {,SRSS }}$} & \\
\hline & & & $\begin{array}{c}\mu \\
(3)\end{array}$ & $\begin{array}{c}\mathrm{COV} \\
(4)\end{array}$ & $\begin{array}{c}\mu \\
(5)\end{array}$ & $\begin{array}{c}\mathrm{COV} \\
(6)\end{array}$ & $\begin{array}{c}\mu \\
(7)\end{array}$ & $\begin{array}{c}\text { COV } \\
(8)\end{array}$ & $\begin{array}{c}\mu \\
(9)\end{array}$ & $\begin{array}{l}\mathrm{COV} \\
(10)\end{array}$ & \\
\hline \multirow{4}{*}{1} & \multirow{4}{*}{ Cortante } & ENT 3 & 1.07 & 0.15 & 1.05 & 0.11 & 1.08 & 0.16 & 1.07 & 0.11 & 40 \\
\hline & & ENT 2 & 1.06 & 0.14 & 1.06 & 0.13 & 1.07 & 0.14 & 1.08 & 0.14 & 40 \\
\hline & & BASE & 1.05 & 0.13 & 1.06 & 0.12 & 1.07 & 0.13 & 1.07 & 0.13 & 40 \\
\hline & & Todos & 1.06 & 0.14 & 1.06 & 0.12 & 1.07 & 0.16 & 1.07 & 0.14 & 120 \\
\hline \multirow{16}{*}{2} & \multirow{6}{*}{$\begin{array}{l}\text { Carga } \\
\text { axial }\end{array}$} & EXT-NS & 0.91 & 0.17 & 0.85 & 0.27 & 0.90 & 0.17 & 0.81 & 0.28 & 40 \\
\hline & & INT-NS & 0.87 & 0.47 & 0.81 & 0.54 & 0.86 & 0.46 & 0.82 & 0.54 & 40 \\
\hline & & GRAV & 0.99 & 0.20 & 1.00 & 0.14 & 0.98 & 0.20 & 0.99 & 0.14 & 40 \\
\hline & & EXT-EW & 0.86 & 0.19 & 0.87 & 0.23 & 0.85 & 0.18 & 0.86 & 0.24 & 40 \\
\hline & & INT-EW & 0.84 & 0.40 & 0.80 & 0.42 & 0.83 & 0.37 & 0.78 & 0.43 & 40 \\
\hline & & Todos & 0.89 & 0.31 & 0.87 & 0.34 & 0.88 & $\mathbf{0 . 3 0}$ & 0.85 & 0.35 & 200 \\
\hline & \multirow{10}{*}{ Cortante } & ENT 9 & 1.10 & 0.14 & 1.07 & 0.12 & 1.13 & 0.13 & 1.09 & 0.10 & 40 \\
\hline & & ENT 8 & 1.12 & 0.14 & 1.04 & 0.12 & 1.15 & 0.12 & 1.05 & 0.10 & 40 \\
\hline & & ENT 7 & 1.11 & 0.09 & 1.04 & 0.11 & 1.14 & 0.09 & 1.06 & 0.10 & 40 \\
\hline & & ENT 6 & 1.09 & 0.10 & 1.05 & 0.09 & 1.12 & 0.09 & 1.06 & 0.08 & 40 \\
\hline & & ENT 5 & 1.09 & 0.10 & 1.06 & 0.11 & 1.12 & 0.09 & 1.07 & 0.10 & 40 \\
\hline & & ENT 4 & 1.09 & 0.10 & 1.07 & 0.11 & 1.12 & 0.10 & 1.07 & 0.10 & 40 \\
\hline & & ENT 3 & 1.11 & 0.10 & 1.08 & 0.12 & 1.14 & 0.10 & 1.09 & 0.11 & 40 \\
\hline & & ENT 2 & 1.11 & 0.12 & 1.06 & 0.12 & 1.14 & 0.11 & 1.07 & 0.11 & 40 \\
\hline & & BASE & 1.11 & 0.11 & 1.09 & 0.12 & 1.14 & 0.10 & 1.09 & 0.11 & 40 \\
\hline & & Todos & 1.10 & 0.10 & 1.06 & 0.10 & 1.13 & 0.11 & 1.07 & 0.10 & 360 \\
\hline
\end{tabular}

Tabla 6. Estadísticas para $\mathrm{R}_{\mathrm{n} 3,30}, \mathrm{R}_{\mathrm{p} 3,30}, \mathrm{R}_{\mathrm{n} 3, \mathrm{SRSS}}$ y $\mathrm{R}_{\mathrm{p} 3, \mathrm{SRSS}}$, carga axial y cortantes de entrepiso, comportamiento inelástico

\begin{tabular}{|c|c|c|c|c|c|c|c|c|c|c|c|}
\hline \multirow{3}{*}{$\begin{array}{l}\text { Modelo } \\
\text { (1) }\end{array}$} & \multirow{3}{*}{\multicolumn{2}{|c|}{$\begin{array}{c}\text { Localización } \\
\text { (2) }\end{array}$}} & \multicolumn{4}{|c|}{ Regla del $30 \%$} & \multicolumn{4}{|c|}{ Regla SRSS } & \multirow{3}{*}{$\begin{array}{c}\text { Tamaño de } \\
\text { la muestra } \\
\text { (11) }\end{array}$} \\
\hline & & & \multicolumn{2}{|c|}{$\mathrm{R}_{\mathrm{n} 3,30}$} & \multicolumn{2}{|c|}{$\mathrm{R}_{\mathrm{p} 3,30}$} & \multicolumn{2}{|c|}{$\mathrm{R}_{\mathrm{n} 3 \mathrm{SRSS}}$} & \multicolumn{2}{|c|}{$\mathrm{R}_{\mathrm{p} 3 \text {,SRSS }}$} & \\
\hline & & & $\begin{array}{c}\mu \\
(3)\end{array}$ & $\begin{array}{c}\mathrm{COV} \\
(4)\end{array}$ & $\begin{array}{c}\mu \\
(5)\end{array}$ & $\begin{array}{c}\mathrm{COV} \\
(6)\end{array}$ & $\begin{array}{c}\mu \\
(7)\end{array}$ & $\begin{array}{c}\mathrm{COV} \\
(8)\end{array}$ & $\begin{array}{c}\mu \\
(9)\end{array}$ & $\begin{array}{l}\mathrm{COV} \\
(10)\end{array}$ & \\
\hline \multirow{6}{*}{1} & \multirow{6}{*}{$\begin{array}{l}\text { Carga } \\
\text { axial }\end{array}$} & EXT-NS & 0.90 & 0.23 & 0.93 & 0.23 & 0.89 & 0.24 & 0.92 & 0.22 & 40 \\
\hline & & INT-NS & 0.87 & 0.23 & 0.89 & 0.19 & 0.83 & 0.23 & 0.86 & 0.19 & 40 \\
\hline & & GRAV & 1.03 & 0.16 & 1.03 & 0.17 & 1.00 & 0.19 & 1.00 & 0.17 & 40 \\
\hline & & EXT-EW & 0.91 & 0.31 & 0.80 & 0.38 & 0.88 & 0.31 & 0.78 & 0.38 & 40 \\
\hline & & INT-EW & 0.88 & 0.28 & 0.93 & 0.23 & 0.84 & 0.28 & 0.90 & 0.24 & 40 \\
\hline & & Todos & 0.92 & 0.24 & 0.92 & 0.24 & 0.89 & 0.24 & 0.89 & 0.24 & 200 \\
\hline
\end{tabular}


Tabla 6. Estadísticas para $\mathrm{R}_{\mathrm{n} 3,30}, \mathrm{R}_{\mathrm{p} 3,30}, \mathrm{R}_{\mathrm{n} 3, \mathrm{SRSS}}$ y $\mathrm{R}_{\mathrm{p} 3, \mathrm{SRSS}}$, carga axial y cortantes de entrepiso, comportamiento inelástico (continuación)

\begin{tabular}{|c|c|c|c|c|c|c|c|c|c|c|c|}
\hline \multirow{3}{*}{$\begin{array}{l}\text { Modelo } \\
\text { (1) }\end{array}$} & \multirow{3}{*}{\multicolumn{2}{|c|}{$\begin{array}{l}\text { Localización } \\
\text { (2) }\end{array}$}} & \multicolumn{4}{|c|}{ Regla del $30 \%$} & \multicolumn{4}{|c|}{ Regla SRSS } & \multirow{3}{*}{$\begin{array}{l}\text { Tamaño de } \\
\text { la muestra } \\
\text { (11) }\end{array}$} \\
\hline & & & \multicolumn{2}{|c|}{$\mathrm{R}_{\mathrm{n} 3,30}$} & \multicolumn{2}{|c|}{$\mathrm{R}_{\mathrm{p} 3,30}$} & \multicolumn{2}{|c|}{$\mathrm{R}_{\mathrm{n} 3, \mathrm{SRSS}}$} & \multicolumn{2}{|c|}{$\mathrm{R}_{\mathrm{p} 3, \mathrm{SRSS}}$} & \\
\hline & & & $\begin{array}{c}\mu \\
(3)\end{array}$ & $\begin{array}{l}\mathrm{COV} \\
(4)\end{array}$ & $\begin{array}{c}\mu \\
(5)\end{array}$ & $\begin{array}{c}\mathrm{COV} \\
\text { (6) }\end{array}$ & $\begin{array}{c}\mu \\
(7)\end{array}$ & $\begin{array}{c}\mathrm{COV} \\
(8)\end{array}$ & $\begin{array}{c}\mu \\
(9)\end{array}$ & $\begin{array}{l}\mathrm{COV} \\
(10)\end{array}$ & \\
\hline \multirow{4}{*}{1} & \multirow{4}{*}{ Cortante } & ENT 3 & 1.07 & 0.17 & 1.06 & 0.09 & 1.08 & 0.17 & 1.08 & 0.09 & 40 \\
\hline & & ENT 2 & 1.06 & 0.13 & 1.08 & 0.09 & 1.08 & 0.14 & 1.10 & 0.09 & 40 \\
\hline & & BASE & 1.04 & 0.14 & 1.08 & 0.08 & 1.05 & 0.14 & 1.09 & 0.08 & 40 \\
\hline & & Todos & 1.06 & 0.14 & 1.07 & 0.08 & 1.07 & 0.15 & 1.09 & 0.09 & 120 \\
\hline \multirow{16}{*}{2} & \multirow{6}{*}{$\begin{array}{l}\text { Carga } \\
\text { axial }\end{array}$} & EXT-NS & 0.92 & 0.25 & 0.84 & 0.24 & 0.90 & 0.22 & 0.84 & 0.26 & 40 \\
\hline & & INT-NS & 0.97 & 0.31 & 0.95 & 0.30 & 0.95 & 0.28 & 0.94 & 0.27 & 40 \\
\hline & & GRAV & 1.02 & 0.14 & 1.01 & 0.19 & 1.01 & 0.16 & 1.02 & 0.18 & 40 \\
\hline & & EXT-EW & 0.95 & 0.27 & 0.84 & 0.24 & 0.93 & 0.25 & 0.83 & 0.24 & 40 \\
\hline & & INT-EW & 0.88 & 0.30 & 0.94 & 0.18 & 0.86 & 0.27 & 0.96 & 0.20 & 40 \\
\hline & & Todos & 0.95 & 0.25 & 0.92 & 0.23 & 0.93 & 0.24 & 0.92 & 0.23 & 200 \\
\hline & \multirow{10}{*}{ Cortante } & ENT 9 & 1.10 & 0.12 & 1.03 & 0.17 & 1.13 & 0.13 & 1.05 & 0.16 & 40 \\
\hline & & ENT 8 & 1.12 & 0.12 & 1.00 & 0.13 & 1.14 & 0.12 & 1.02 & 0.13 & 40 \\
\hline & & ENT 7 & 1.12 & 0.08 & 1.03 & 0.12 & 1.14 & 0.09 & 1.04 & 0.12 & 40 \\
\hline & & ENT 6 & 1.09 & 0.09 & 1.01 & 0.18 & 1.12 & 0.09 & 1.02 & 0.17 & 40 \\
\hline & & ENT 5 & 1.09 & 0.09 & 1.04 & 0.13 & 1.12 & 0.09 & 1.04 & 0.13 & 40 \\
\hline & & ENT 4 & 1.09 & 0.09 & 1.02 & 0.15 & 1.12 & 0.10 & 1.03 & 0.15 & 40 \\
\hline & & ENT 3 & 1.11 & 0.09 & 1.03 & 0.18 & 1.14 & 0.09 & 1.03 & 0.18 & 40 \\
\hline & & ENT 2 & 1.12 & 0.10 & 1.01 & 0.18 & 1.15 & 0.10 & 1.02 & 0.18 & 40 \\
\hline & & BASE & 1.12 & 0.09 & 1.03 & 0.17 & 1.15 & 0.09 & 1.04 & 0.17 & 40 \\
\hline & & Todos & 1.11 & 0.10 & 1.02 & 0.16 & 1.14 & 0.11 & 1.03 & 0.16 & 360 \\
\hline
\end{tabular}

\section{Precisión de las reglas para parámetros de respuesta múltiples}

Hasta el momento se ha discutido la precisión de las reglas del 30\% y SRSS en la estimación de la respuesta combinada para parámetros de respuesta individuales. Sin embargo, lo que se tiene en general en un estado de carga, es la presencia de varías fuerzas actuando simultáneamente (carga axial, cortante, momentos flexionantes y torsionantes). Por lo anterior, la estimación de la precisión de las reglas para el caso más realista de parámetros de respuesta combinados es importante y se aborda a continuación. La consideración de dichas fuerzas actuando simultáneamente se realiza mediante las ecuaciones de interacción de las Specification for Structural Steel Buildings del American Institute of Steel Construction (AISC 2005). En su capítulo H titulado Design of members for combined forces and torsion, se establecen dichas ecuaciones para estimar la respuesta de los elementos estructurales sometidos a las combinaciones de fuerzas que en ellos actúan.

Para el caso de elementos con uno o dos ejes de simetría sujetos a carga axial y flexión, se especifica: 


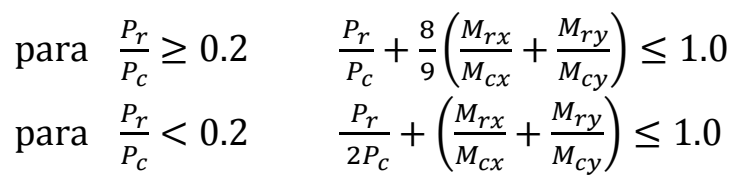

dónde:

$P_{r}=$ resistencia axial requerida (fuerza)

$P_{c}=$ resistencia axial disponible (fuerza)

$M_{r}=$ resistencia a flexión requerida (fuerza - longitud)

$M_{c}=$ resistencia a flexión disponible (fuerza - longitud)

$x=$ subíndice relacionado a la flexión alrededor del eje fuerte del elemento

$y$ = subíndice relacionado a la flexión alrededor del eje débil del elemento

De acuerdo a la Sección B3.3 de las especificaciones, $P_{r}$ y $M_{r}$ se determinan de acuerdo a las combinaciones de carga LRFD, $P_{c}=\emptyset_{c} P_{n}$ y $M_{c}=\emptyset_{b} M_{n}$, donde:

$\emptyset_{c}=$ factor de reducción de resistencia a compresión $=0.90$

$\emptyset_{b}=$ factor de reducción de resistencia a flexión $=0.90$

$P_{n}=$ resistencia axial nominal (fuerza)

$M_{n}=$ resistencia a flexión nominal (fuerza - longitud)

Se debe notar que $P_{c}, M_{c x}$ y $M_{c y}$ permanecerán constantes para cada elemento estructural considerado, solo las resistencias requeridas $P_{r}, M_{r x}$ y $M_{r y}$ serán diferentes para cada sismo aplicado. Las respuestas de referencia $\mathrm{R}_{\mathrm{n} 2}, \mathrm{R}_{\mathrm{p} 2}, \mathrm{R}_{\mathrm{n} 3}$ y $\mathrm{R}_{\mathrm{p} 3}$ representarán lo mismo que antes, la única diferencia es que ahora se estiman usando las ecuaciones de interacción. Por ejemplo, $\mathrm{R}_{\mathrm{n} 3}$ para un elemento dado se calcula con la Ecuación (5) o (6) donde los valores de $\mathrm{P}_{\mathrm{r}}, \mathrm{M}_{\mathrm{rx}}$ y $\mathrm{M}_{\mathrm{ry}}$ se obtienen de la mayor de las respuestas de los Casos de Carga 5 y 6. Similarmente, $\mathrm{R}_{\mathrm{p} 3}$ se obtendrá de la mayor de las respuestas de los Casos de Carga 7 y 8 . La respuesta combinada se calcula igual que antes, solo que ahora cada uno de los términos en las ecuaciones de combinación se obtiene con las ecuaciones de interacción. Por ejemplo, para el caso de componentes principales, en las expresiones ${ }^{11} X_{p}+0.30{ }^{11} Y_{p}+0.30{ }^{11} Z_{p}, 0.30{ }^{11} X_{p}+{ }^{11} Y_{p}+$ $0.30{ }^{11} Z_{p}$ y $0.30{ }^{11} X_{p}+0.30{ }^{11} Y_{p}+{ }^{11} Z_{p}$, los parámetros $X_{p}, Y_{p}$ y $Z_{p}$ se estiman con la ecuación (5) o (6) sustituyendo en ellas los valores de $P_{r}, M_{r x} \mathrm{y} M_{r y}$, obtenidas del análisis con el Caso de Carga 11. De esta

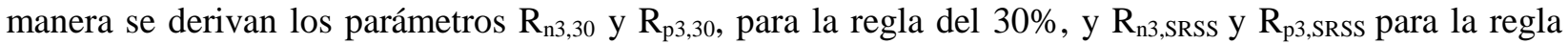
SRSS. Para el caso en que solo se consideren las componentes horizontales, los parámetros $R_{n 2,30}, R_{\mathrm{p} 2,30}$, $\mathrm{R}_{\mathrm{n} 2, \mathrm{SRSS}}$ y $\mathrm{R}_{\mathrm{p} 2 \text {,SRSS }}$ se obtienen siguiendo un procedimiento similar al mencionado.

Las estadísticas para ambos modelos y reglas de combinación se presentan en la Tabla 7 para comportamiento elástico. Los resultados indican que, en promedio, ambas reglas subestiman la respuesta combinada alrededor de $15 \%$ y la incertidumbre asociada es alrededor de $15 \%$. No se observan diferencias significativas entre ambas reglas de combinación ni entre componentes normales y principales. Los resultados para comportamiento inelástico siguen la misma tendencia, la única observación adicional es que los niveles de subestimación y de incertidumbre se incrementan ligeramente. Además, se puede observar que las estadísticas son prácticamente las mismas para dos y tres componentes, esto se debe a que la componente vertical no produce momentos significativos en las columnas y a que los valores de las ecuaciones (5) y (6) se definen principalmente por la magnitud de los momentos. 
Tabla 7. Estadísticas de la precisión de las reglas de combinación, parámetros de respuesta múltiples, comportamiento elástico, componentes normales y principales

\begin{tabular}{|c|c|c|c|c|c|c|c|c|c|c|c|c|c|c|c|c|c|c|}
\hline \multirow{3}{*}{ Modelo } & \multirow{3}{*}{ Localización } & \multicolumn{8}{|c|}{ Regla del $30 \%$} & \multicolumn{8}{|c|}{ Regla SRSS } & \multirow{3}{*}{$\begin{array}{l}\text { Tamaño } \\
\text { de la } \\
\text { muestra }\end{array}$} \\
\hline & & \multicolumn{2}{|c|}{$\mathrm{R}_{\mathrm{n} 2,30}$} & \multicolumn{2}{|c|}{$\mathrm{R}_{\mathrm{n} 3,30}$} & \multicolumn{2}{|c|}{$\mathrm{R}_{\mathrm{p} 2,30}$} & \multicolumn{2}{|c|}{$\mathrm{R}_{\mathrm{p} 3,30}$} & \multicolumn{2}{|c|}{$\mathrm{R}_{\mathrm{n} 2, \mathrm{SRSS}}$} & \multicolumn{2}{|c|}{$\mathrm{R}_{\mathrm{n} 3, \mathrm{SRSS}}$} & \multicolumn{2}{|c|}{$\mathrm{R}_{\mathrm{p} 2 \text {,SRSS }}$} & \multicolumn{2}{|c|}{$\mathrm{R}_{\mathrm{p} 3, \mathrm{SRSS}}$} & \\
\hline & & $\mu$ & $\mathrm{COV}$ & $\mu$ & $\mathrm{COV}$ & $\mu$ & $\mathrm{COV}$ & $\mu$ & $\mathrm{COV}$ & $\mu$ & $\mathrm{COV}$ & $\mu$ & $\mathrm{COV}$ & $\mu$ & $\mathrm{COV}$ & $\mu$ & $\mathrm{COV}$ & \\
\hline \multirow{6}{*}{1} & EXT-NS & 0.86 & 0.12 & 0.86 & 0.12 & 0.85 & 0.11 & 0.85 & 0.1 & 0.86 & 0.12 & 0.86 & 0.12 & 0.85 & 0.11 & 0.85 & 0.11 & 40 \\
\hline & INT-NS & 0.87 & 0.11 & 0.87 & 0.11 & 0.87 & 0.09 & 0.87 & 0.1 & 0.86 & 0.11 & 0.86 & 0.11 & 0.86 & 0.09 & 0.86 & 0.09 & 40 \\
\hline & GRAV & 0.87 & 0.19 & 0.9 & 0.18 & 0.89 & 0.14 & 0.9 & 0.14 & 0.86 & 0.19 & 0.88 & 0.18 & 0.88 & 0.14 & 0.89 & 0.14 & 40 \\
\hline & EXT-EW & 0.86 & 0.2 & 0.86 & 0.2 & 0.86 & 0.18 & 0.86 & 0.17 & 0.86 & 0.2 & 0.86 & 0.2 & 0.86 & 0.18 & 0.86 & 0.18 & 40 \\
\hline & INT-EW & 0.87 & 0.18 & 0.87 & 0.18 & 0.87 & 0.15 & 0.87 & 0.15 & 0.86 & 0.18 & 0.86 & 0.18 & 0.86 & 0.16 & 0.86 & 0.16 & 40 \\
\hline & odos & 0.87 & 0.16 & 0.87 & 0.16 & 0.87 & 0.14 & 0.87 & 0.14 & 0.86 & 0.16 & 0.87 & 0.16 & 0.86 & 0.14 & 0.86 & 0.14 & 200 \\
\hline & EXT-NS & 0.87 & 0.12 & 0.87 & 0.12 & 0.82 & 0.14 & 0.82 & 0.14 & 0.87 & 0.12 & 0.87 & 0.12 & 0.82 & 0.14 & 0.82 & 0.14 & 40 \\
\hline & INT-NS & 0.87 & 0.12 & 0.87 & 0.12 & 0.82 & 0.15 & 0.82 & 0.15 & 0.87 & 0.12 & 0.87 & 0.12 & 0.82 & 0.15 & 0.82 & 0.15 & 40 \\
\hline & GRAV & 0.88 & 0.14 & 0.89 & 0.13 & 0.83 & 0.18 & 0.84 & 0.18 & 0.88 & 0.14 & 0.88 & 0.14 & 0.82 & 0.18 & 0.83 & 0.18 & 40 \\
\hline & EXT-EW & 0.87 & 0.17 & 0.87 & 0.17 & 0.83 & 0.22 & 0.83 & 0.22 & 0.87 & 0.17 & 0.87 & 0.17 & 0.82 & 0.22 & 0.82 & 0.22 & 40 \\
\hline & INT-EW & 0.87 & 0.17 & 0.87 & 0.17 & 0.82 & 0.22 & 0.82 & 0.22 & 0.87 & 0.17 & 0.87 & 0.17 & 0.82 & 0.22 & 0.82 & 0.22 & 40 \\
\hline & Todos & 0.87 & 0.14 & 0.87 & 0.14 & 0.82 & 0.18 & 0.83 & 0.18 & 0.87 & 0.14 & 0.87 & 0.14 & 0.82 & 0.19 & 0.82 & 0.18 & 200 \\
\hline
\end{tabular}

Con base en los resultados anteriores se pueden concluir que, en general, ambas reglas subestiman la carga axial en las columnas de los modelos considerados alrededor de $15 \%$ para componentes normales y principales, y la incertidumbre asociada a dicha subestimación (COV) es alrededor de un 30\%. Sin embargo, estas sobreestiman razonablemente los cortantes de entrepiso. Por otra parte, se observa que los niveles de subestimación y de incertidumbre se incrementan cuando se considera comportamiento estructural inelástico. No se observan diferencias significativas entre ambas reglas de combinación, no obstante, estas son ligeramente más precisas en estimar la respuesta combinada en términos de carga axial cuando se consideran las tres componentes de los terremotos que cuando se consideran solo las componentes horizontales. Para parámetros de respuesta múltiples, la respuesta combinada también se subestima alrededor de $15 \%$. Estos resultados indican que para SVGL hay un cierto grado de correlación entre los efectos individuales de las componentes de los terremotos, incluso para componentes no correlacionadas (principales).

\section{Correlación de los efectos individuales}

La hipótesis básica para la aplicación de la regla de combinación SRSS es que las componentes de aceleración de los sismos no están correlacionadas. Se asume implícitamente que si no hay correlación entre los acelerogramas, los correspondientes efectos también estarán no correlacionados. Si esto se cumpliera, la regla SRSS daría la solución exacta y la regla del 30\% daría una solución muy cercana a la de la SRSS. En esta sección, se estudia la influencia que tiene la correlación de las componentes de los terremotos así como la de los correspondientes efectos individuales en la precisión de las reglas de combinación. Para ello se calcularon los coeficientes de correlación $(\rho)$ de los efectos individuales para los Modelos 1 y 2, para componentes normales y principales, para comportamiento elástico e inelástico y para parámetros de respuesta colineales (carga axial) y no colineales (cortante basal). Solo se muestran los resultados para las columnas del Modelo 1 y comportamiento elástico. No obstante, los resultados para comportamiento inelástico y aquellos correspondientes al Modelo 2 son muy similares. 
En la Columna (2) de la Tabla 8 se muestran los coeficientes de correlación entre las componentes normales de los 20 terremotos usados en este estudio, los cuales se denotan por $\rho_{\text {No. }}$ Se observa que en algunos casos las componentes pueden ser altamente correlacionadas, se presentan valores cercanos a 0.50. Los coeficientes correspondientes a las componentes principales son obviamente cero. Los coeficientes de correlación ( $\rho$ ) de los efectos individuales se dan en las columnas (3) a la (8) de la Tabla 8 y en las columnas (2) a la (7) de la Tabla 9, para componentes normales y principales, respectivamente. Los resultados en las tablas indican que los valores de los coeficientes varían de un sismo a otro y de un elemento a otro sin mostrar tendencia alguna. La mayoría de los valores son despreciables (menores a 0.25), sin embargo, para algunos casos hay valores considerables (mayores a 0.50), incluso para componentes principales. De los resultados de la Tabla 8 y de la Figura 2, se observa que valores considerables de $\rho$ no siempre están relacionados a una estimación imprecisa de la respuesta combinada y que las reglas no siempre son precisas para valores pequeños de $\rho$. Por ejemplo, de la Tabla 8 se puede observar que para el Sismo 1, los coeficientes de correlación para carga axial en las columnas EXT-NS, INT-NS, GRAV y EXT-EW son considerables (mayores a 0.40), sin embargo, de la Figura 2a se observa que la regla de combinación del $30 \%$ estima adecuadamente la respuesta combinada para estas columnas. Por otra parte, para la columna INT-EW y el mismo sismo se tienen efectos no correlacionados, sin embargo, la respuesta se subestima alrededor de $30 \%$.

Tabla 8. Coeficientes de correlación ( $\rho)$, comportamiento elástico, Modelo 1, componentes normales

\begin{tabular}{cccccccc}
\hline $\begin{array}{c}\text { Sismo } \\
(1)\end{array}$ & $\begin{array}{c}\rho_{\text {NO }} \\
(2)\end{array}$ & $\begin{array}{c}\text { EXT-NS } \\
(3)\end{array}$ & $\begin{array}{c}\text { INT-NS } \\
(4)\end{array}$ & $\begin{array}{c}\text { GRAV } \\
(5)\end{array}$ & $\begin{array}{c}\text { EXT-EW } \\
(6)\end{array}$ & $\begin{array}{c}\text { INT-EW } \\
(7)\end{array}$ & $\begin{array}{c}\text { CORTANTE } \\
(8)\end{array}$ \\
\hline 1 & -0.06 & 0.54 & 0.41 & 0.60 & 0.64 & -0.02 & 0.67 \\
2 & 0.34 & 0.72 & 0.49 & 0.71 & 0.73 & 0.66 & 0.79 \\
3 & 0.07 & -0.15 & -0.12 & -0.14 & -0.14 & -0.13 & -0.22 \\
4 & 0.08 & -0.08 & -0.09 & -0.07 & -0.04 & -0.09 & -0.11 \\
5 & 0.35 & 0.47 & 0.38 & 0.48 & 0.49 & 0.42 & 0.37 \\
6 & 0.41 & 0.56 & 0.28 & 0.53 & 0.55 & 0.28 & 0.50 \\
7 & 0.44 & 0.38 & 0.42 & 0.39 & 0.36 & 0.31 & 0.54 \\
8 & 0.41 & 0.36 & 0.22 & 0.34 & 0.31 & 0.25 & 0.47 \\
9 & 0.35 & 0.08 & 0.18 & 0.10 & 0.10 & -0.04 & 0.23 \\
10 & 0.23 & -0.04 & -0.02 & -0.04 & -0.04 & -0.05 & 0.10 \\
11 & 0.25 & -0.01 & -0.12 & -0.03 & -0.02 & -0.05 & 0.04 \\
12 & 0.34 & 0.01 & 0.01 & 0.01 & 0.02 & -0.08 & 0.11 \\
13 & 0.02 & -0.11 & -0.07 & -0.09 & -0.06 & -0.12 & -0.14 \\
14 & 0.25 & 0.22 & -0.01 & 0.21 & 0.22 & 0.18 & 0.38 \\
15 & 0.24 & 0.03 & 0.12 & 0.06 & 0.08 & 0.03 & 0.12 \\
16 & 0.09 & -0.11 & 0.00 & -0.09 & -0.10 & -0.01 & -0.06 \\
17 & 0.19 & 0.28 & 0.05 & 0.25 & 0.21 & 0.33 & 0.47 \\
18 & 0.15 & 0.13 & 0.20 & 0.17 & 0.13 & 0.16 & 0.32 \\
19 & 0.22 & 0.34 & 0.28 & 0.36 & 0.37 & 0.22 & 0.50 \\
20 & 0.04 & -0.13 & 0.01 & -0.11 & -0.11 & -0.06 & -0.23 \\
\hline
\end{tabular}


Tabla 9. Coeficientes de correlación ( $\rho$ ), comportamiento elástico, Modelo 1, componentes principales

\begin{tabular}{ccccccc}
\hline $\begin{array}{c}\text { Sismo } \\
(1)\end{array}$ & $\begin{array}{c}\text { EXT-NS } \\
(2)\end{array}$ & $\begin{array}{c}\text { INT-NS } \\
(3)\end{array}$ & $\begin{array}{c}\text { GRAV } \\
(4)\end{array}$ & $\begin{array}{c}\text { EXT-EW } \\
(5\end{array}$ & $\begin{array}{c}\text { INT-EW } \\
(6)\end{array}$ & $\begin{array}{c}\text { CORTANTE } \\
(7)\end{array}$ \\
\hline 1 & 0.62 & 0.38 & 0.69 & 0.74 & -0.05 & 0.72 \\
2 & 0.24 & 0.09 & 0.21 & 0.36 & -0.34 & 0.35 \\
3 & -0.03 & 0.17 & 0.02 & 0.06 & -0.22 & 0.07 \\
4 & 0.04 & 0.14 & 0.05 & 0.01 & 0.14 & 0.12 \\
5 & 0.20 & 0.26 & 0.23 & 0.18 & 0.18 & 0.39 \\
6 & -0.02 & 0.07 & 0.00 & 0.00 & 0.01 & 0.13 \\
7 & 0.18 & 0.22 & 0.20 & 0.22 & -0.08 & 0.37 \\
8 & 0.18 & 0.15 & 0.17 & 0.27 & -0.20 & 0.40 \\
9 & 0.12 & 0.23 & 0.16 & 0.15 & 0.22 & 0.33 \\
10 & 0.16 & 0.25 & 0.19 & 0.15 & 0.24 & 0.37 \\
11 & 0.75 & 0.58 & 0.75 & 0.74 & -0.05 & 0.50 \\
12 & 0.51 & 0.66 & 0.53 & 0.49 & -0.23 & 0.52 \\
13 & -0.05 & -0.01 & -0.05 & 0.03 & -0.24 & 0.03 \\
14 & 0.50 & 0.32 & 0.43 & 0.34 & 0.33 & 0.62 \\
15 & 0.24 & 0.22 & 0.25 & 0.24 & 0.06 & 0.21 \\
16 & 0.39 & 0.16 & 0.36 & 0.47 & -0.30 & 0.33 \\
17 & 0.13 & 0.07 & 0.13 & 0.26 & -0.29 & 0.35 \\
18 & 0.42 & 0.12 & 0.36 & 0.26 & 0.42 & 0.40 \\
19 & 0.11 & 0.11 & 0.12 & 0.14 & -0.06 & 0.29 \\
20 & 0.45 & 0.34 & 0.50 & 0.55 & 0.00 & 0.59 \\
\hline
\end{tabular}

De los resultados de las secciones anteriores se observa que hay varios factores que intervienen en la precisión de las reglas de combinación, tales como el grado de correlación de las componentes de los terremotos y de sus correspondientes efectos individuales, la localización del elemento considerado, el nivel de deformación estructural y el parámetro de respuesta al cual se aplican. En este último caso, claramente se observa que la precisión de las reglas es muy diferente para parámetros de respuesta colineales (carga axial en columnas) y no colineales (cortante basal). Para el caso de estructuras simétricas, como los considerados en este estudio, para una dirección horizontal específica, el cortante basal no se afecta por la componente que actúa en la dirección perpendicular, sin embargo, la carga axial en columnas se afecta por la acción de las tres componentes. Las contribuciones de cada componente a la carga axial de un columna particular durante algunos periodos de tiempo puede estar en fase una con otra, pero para otros periodos de tiempo pueden estar desfasados. Este situación no ocurre para el caso de cortante basal. Además la carga axial también depende de la distancia de su ubicación al centro de rigidez de la estructura. Por lo anterior, los reglamentos deben ser más específicos con respecto a la aplicación de las reglas mencionadas. 


\section{ORIENTACIÓN CRÍTICA DE LAS COMPONENTES DE LOS TERREMOTOS}

\section{Carga axial}

Otro de los objetivos principales de este trabajo es estudiar la orientación crítica de las componentes de los terremotos. Para ello, las componentes normales horizontales de los 20 registros sísmicos, cuyas características se describen en la Tabla 2, se rotan de 0 a 90 grados, con incrementos de 5 grados. Después, se introduce el parámetro $\mathrm{R}$, que se define como la razón de la respuesta producida por las componentes normales (o rotadas a cierto ángulo de incidencia) y aquella producida por las componentes principales. Por lo tanto, valores de R mayores a 1.0 indican que la respuesta crítica se produce por las componentes normales o rotadas, por otra parte, valores menores a 1.0 indican que la respuesta crítica se produce por las componentes principales. Los valores de $\mathrm{R}$ se calculan para los Modelos 1 y 2, para comportamiento elástico e inelástico y para parámetros de respuesta colineales (carga axial) y nocolineales (cortantes de entrepiso y basal).

Los valores calculados de R para carga axial, se presentan en la Figura 6, para el Modelo 1 y comportamiento elástico. Solo se muestran los resultados para algunos sismos. Se observa que el parámetro $\mathrm{R}$ varía con el ángulo de incidencia de las componentes, con la localización del elemento y con el terremoto considerado, sin mostrar tendencia alguna. Se observa también que, para un terremoto dado, el ángulo de incidencia correspondiente al valor máximo de $\mathrm{R}$ (ángulo crítico) es, en general, diferente para cada elemento considerado. La observación más importante que puede hacerse es que, en muchos casos, la respuesta es mayor a la producida por las componentes principales. Se observan valores de $\mathrm{R}$ cercanos a 1.30. Para el caso de las columnas que pertenecen a un marco de gravedad (GRAV), la variación de $\mathrm{R}$ con el ángulo de incidencia es mucho menor que para las columnas que pertenecen a los marcos resistentes a momento. La razón de esto es que el efecto de las componentes horizontales en la carga axial de estas columnas es mucho menor.

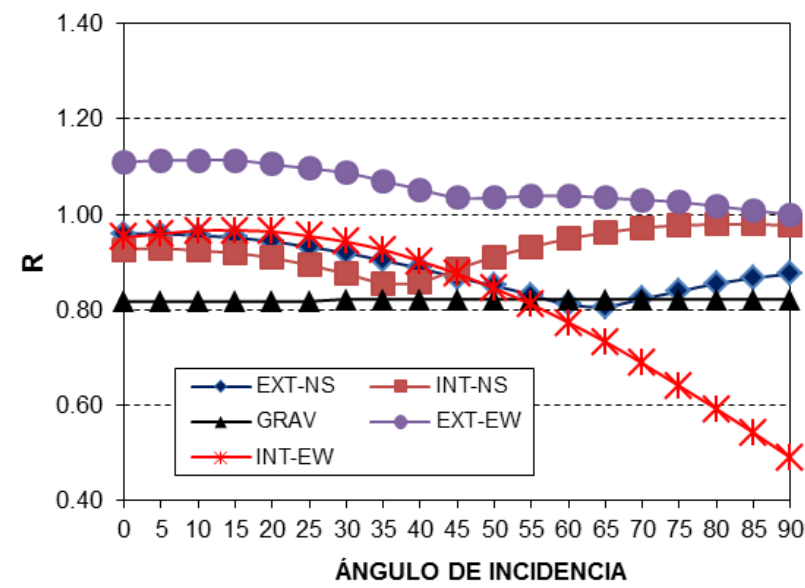

a) Sismo 03

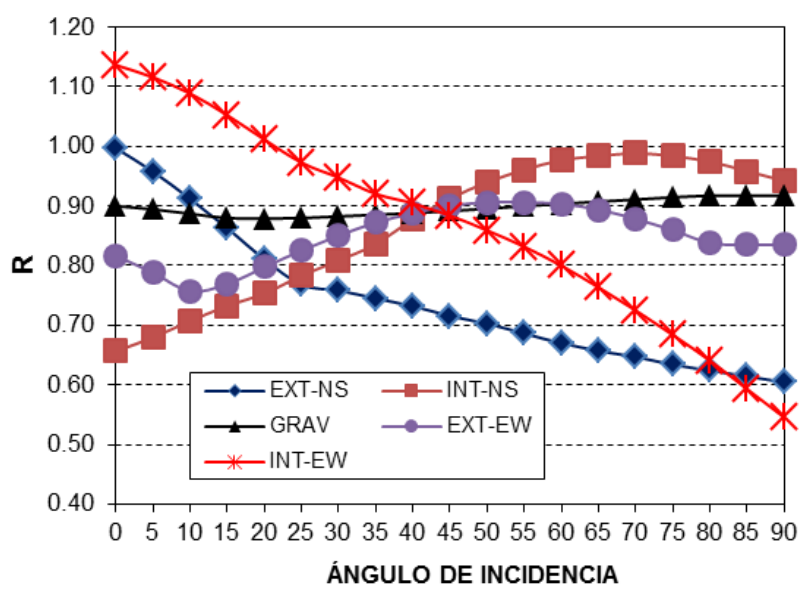

b) Sismo 09 


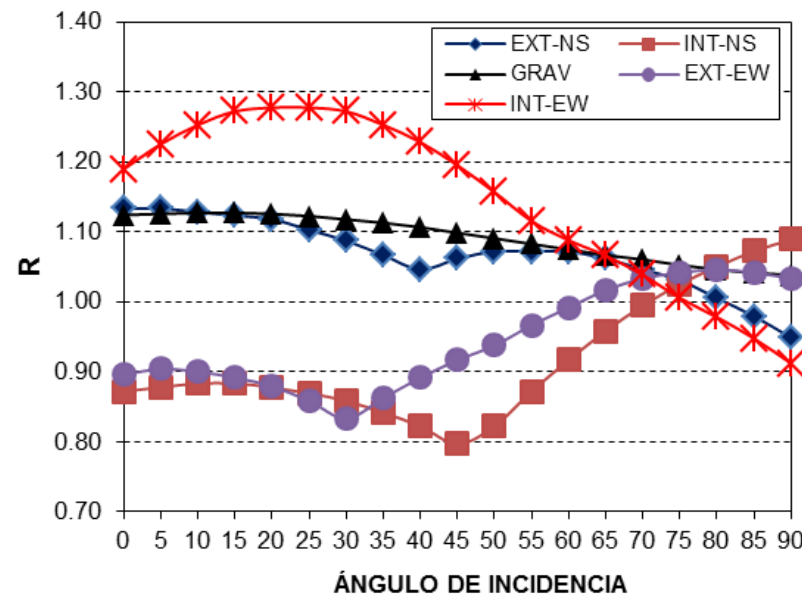

c) Sismo 14

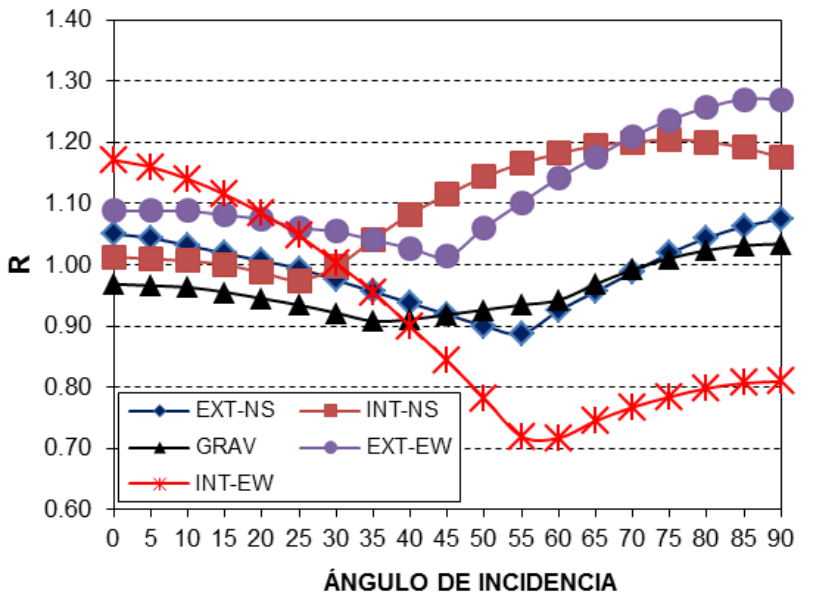

d) Sismo 20

Figura 6. Parámetro R, carga axial, comportamiento elástico, Modelo 1

En la Tabla 10 se dan los valores máximos de $\mathrm{R}$ para cada sismo, así como las estadísticas correspondientes para ambos modelos. De los resultados de la tabla se puede observar que el valor máximo de $\mathrm{R}$ varía de un sismo a otro y de un elemento a otro sin mostrar tendencia alguna, con valores que van de 0.58 a 2.03 . La media y el COV de R, considerando los dos modelos, todas las columnas y todos los registros sísmicos, son 1.04 y 0.21 , respectivamente. Esto indica que la respuesta crítica es muy cercana a la producida por las componentes principales.

Tabla 10. Valores máximos de R, carga axial, comportamiento elástico

\begin{tabular}{|c|c|c|c|c|c|c|c|c|c|c|}
\hline \multirow[b]{2}{*}{ Sismo } & \multicolumn{5}{|c|}{ Modelo 1} & \multicolumn{5}{|c|}{ Modelo 2} \\
\hline & EXT-NS & INT-NS & GRAV & $\begin{array}{l}\text { EXT- } \\
\text { EW }\end{array}$ & INT-EW & EXT-NS & INT-NS & GRAV & $\begin{array}{c}\text { EXT- } \\
\text { EW }\end{array}$ & INT-EW \\
\hline 1 & 0.99 & 1.06 & 1.02 & 1.01 & 1.28 & 1.02 & 1.03 & 1.08 & 0.67 & 1.00 \\
\hline 2 & 0.97 & 1.01 & 0.96 & 1.12 & 0.63 & 1.11 & 0.96 & 0.80 & 1.83 & 0.97 \\
\hline 3 & 0.96 & 0.98 & 0.82 & 1.11 & 0.97 & 0.78 & 0.80 & 0.58 & 1.34 & 0.81 \\
\hline 4 & 1.07 & 0.94 & 1.35 & 0.89 & 0.93 & 0.69 & 0.87 & 0.99 & 0.83 & 0.87 \\
\hline 5 & 1.03 & 1.04 & 1.03 & 1.15 & 1.03 & 0.94 & 1.00 & 0.90 & 1.63 & 1.00 \\
\hline 6 & 0.83 & 0.97 & 0.87 & 0.99 & 0.97 & 1.13 & 1.01 & 1.00 & 1.76 & 1.02 \\
\hline 7 & 0.80 & 0.97 & 0.93 & 0.97 & 0.95 & 1.05 & 1.02 & 1.02 & 1.43 & 1.01 \\
\hline 8 & 0.92 & 1.00 & 0.96 & 1.05 & 0.61 & 1.01 & 0.99 & 0.99 & 2.03 & 1.01 \\
\hline 9 & 1.00 & 0.99 & 0.92 & 0.91 & 1.14 & 0.64 & 1.06 & 1.25 & 1.04 & 1.07 \\
\hline 10 & 1.05 & 1.04 & 1.02 & 1.04 & 1.04 & 1.09 & 1.00 & 0.99 & 1.26 & 1.00 \\
\hline 11 & 1.24 & 1.03 & 1.08 & 1.06 & 1.20 & 0.65 & 1.17 & 1.14 & 0.68 & 1.21 \\
\hline 12 & 1.19 & 1.15 & 0.95 & 1.14 & 0.70 & 0.80 & 1.09 & 0.97 & 1.35 & 1.13 \\
\hline 13 & 1.01 & 1.00 & 0.99 & 1.01 & 0.76 & 1.01 & 0.98 & 1.06 & 1.89 & 0.98 \\
\hline 14 & 1.13 & 1.09 & 1.13 & 1.05 & 1.28 & 0.65 & 0.98 & 0.98 & 1.19 & 1.00 \\
\hline 15 & 0.88 & 1.18 & 0.87 & 1.06 & 0.79 & 0.85 & 1.03 & 0.93 & 1.45 & 1.06 \\
\hline 16 & 0.97 & 1.05 & 1.08 & 1.07 & 0.84 & 1.05 & 1.04 & 0.96 & 1.82 & 1.06 \\
\hline 17 & 0.93 & 1.04 & 0.95 & 1.13 & 0.61 & 1.07 & 0.99 & 0.88 & 1.68 & 1.00 \\
\hline
\end{tabular}


Tabla 10. Valores máximos de R, carga axial, comportamiento elástico (continuación)

\begin{tabular}{|c|c|c|c|c|c|c|c|c|c|c|}
\hline \multirow[b]{2}{*}{ Sismo } & \multicolumn{5}{|c|}{ Modelo 1} & \multicolumn{5}{|c|}{ Modelo 2} \\
\hline & EXT-NS & INT-NS & GRAV & $\begin{array}{c}\text { EXT- } \\
\text { EW }\end{array}$ & INT-EW & EXT-NS & INT-NS & GRAV & $\begin{array}{c}\text { EXT- } \\
\text { EW }\end{array}$ & INT-EW \\
\hline 18 & 1.04 & 1.11 & 1.08 & 1.29 & 0.80 & 1.08 & 0.93 & 1.75 & 1.14 & 0.97 \\
\hline 19 & 1.01 & 0.99 & 1.00 & 1.16 & 0.92 & 1.10 & 1.01 & 0.83 & 1.64 & 1.03 \\
\hline 20 & 1.08 & 1.20 & 1.03 & 1.27 & 1.17 & 1.22 & 1.02 & 1.01 & 1.49 & 1.07 \\
\hline$\mu$ & 1.01 & 1.04 & 1.00 & 1.07 & 0.93 & 0.95 & 1.00 & 1.01 & 1.41 & 1.01 \\
\hline \multirow[t]{2}{*}{$\mathrm{COV}$} & 0.11 & 0.07 & 0.11 & 0.10 & 0.23 & 0.19 & 0.08 & 0.22 & 0.28 & 0.08 \\
\hline & \multicolumn{5}{|c|}{$\mu($ Todos $)=1.04$} & \multicolumn{4}{|c|}{$\operatorname{COV}($ Todos $)=0.21$} & \\
\hline
\end{tabular}

Como se comentó anteriormente, los 20 registros sísmicos se escalaron en términos de $S_{a}\left(T_{1}\right)$, y después para estudiar el efecto del comportamiento inelástico, estos se escalaron gradualmente para lograr fluencia significativa en los modelos. Los resultados del Modelo 1 para los sismos 03, 09, 14 y 20 se muestran en la Figura 7. Se observa que, al igual que para comportamiento elástico, el parámetro R varía de un sismo a otro, de un elemento a otro y de un ángulo de incidencia a otro sin mostrar ninguna tendencia. En este caso, la respuesta es mucho más sensible al ángulo de incidencia que para comportamiento elástico. Para las columnas de los marcos de gravedad, los valores de $\mathrm{R}$ son prácticamente los mismos para todos los ángulos de incidencia. El ángulo crítico es, en general, diferente para comportamiento elástico e inelástico. La observación más importante es que la respuesta crítica es mucho mayor a aquella producida por las componentes principales, se observan valores de R cercanos a 3 en algunos casos.

Las estadísticas de los valores máximos de $\mathrm{R}$ para comportamiento inelástico se dan en la Tabla 11. Los resultados indican que los valores de $\mathrm{R}$ varían de un sismo a otro y de elemento a otro sin mostrar tendencias, estos van de 0.42 a 2.94. En comparación al caso elástico se tienen valores de $\mathrm{R}$ considerablemente mayores para comportamiento inelástico. La incertidumbre en la estimación también se incrementa de manera significativa. Los resultados indican que, en promedio, la carga axial crítica puede ser hasta un $30 \%$ mayor a aquella producida por las componentes principales.

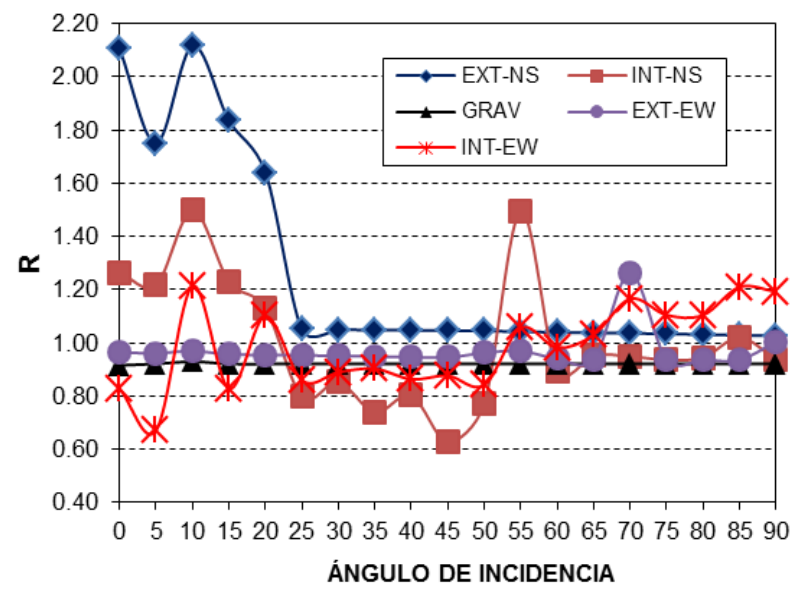

a) Sismo 03

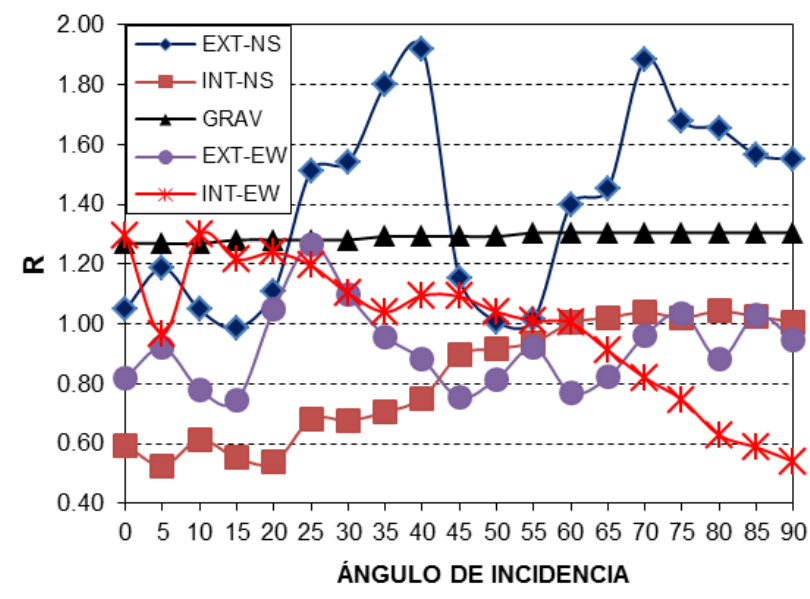

b) Sismo 09 


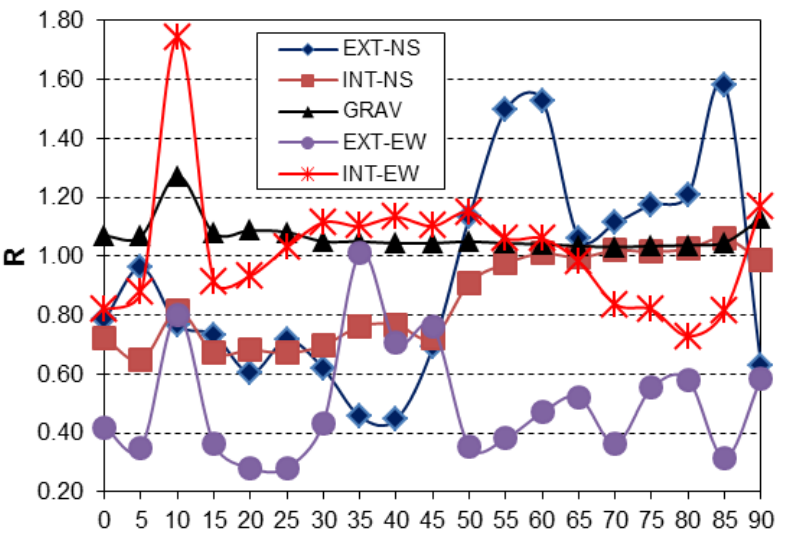

ÁNGULO DE INCIDENCIA

c) Sismo 14

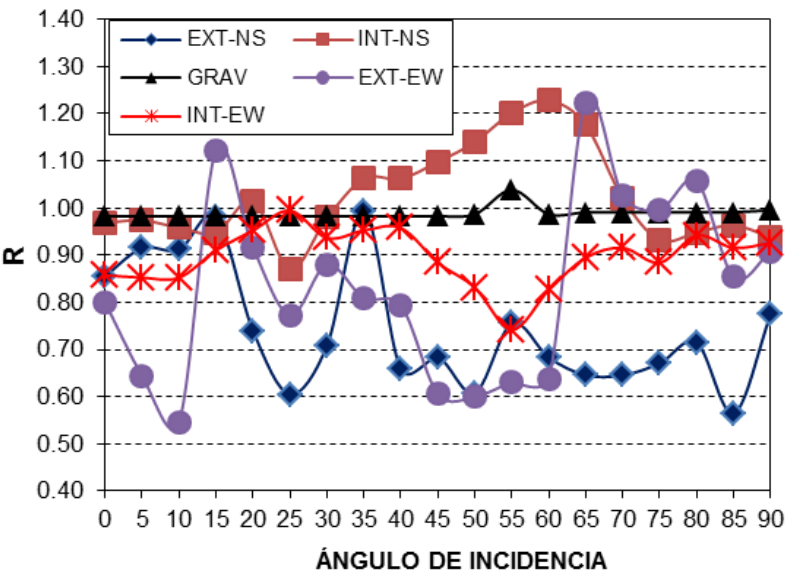

d) Sismo 20

Figura 7. Parámetro R, carga axial, comportamiento inelástico, Modelo 1

Tabla 11. Valores máximos de R, carga axial, comportamiento inelástico

\begin{tabular}{|c|c|c|c|c|c|c|c|c|c|c|}
\hline \multirow[b]{2}{*}{ Sismo } & \multicolumn{5}{|c|}{ Modelo 1} & \multicolumn{5}{|c|}{ Modelo 2} \\
\hline & EXT-NS & INT-NS & GRAV & $\begin{array}{c}\text { EXT- } \\
\text { EW }\end{array}$ & INT-EW & EXT-NS & INT-NS & GRAV & $\begin{array}{l}\text { EXT- } \\
\text { EW }\end{array}$ & INT-EW \\
\hline 1 & 1.63 & 1.09 & 1.05 & 1.30 & 1.01 & 1.17 & 1.05 & 1.42 & 1.10 & 1.01 \\
\hline 2 & 1.08 & 0.89 & 1.29 & 1.16 & 1.06 & 1.02 & 1.29 & 1.15 & 0.93 & 1.02 \\
\hline 3 & 2.12 & 1.50 & 0.93 & 1.26 & 1.21 & 1.25 & 0.74 & 0.87 & 0.94 & 0.74 \\
\hline 4 & 1.35 & 1.03 & 1.39 & 1.52 & 1.12 & 1.03 & 1.52 & 1.44 & 0.88 & 1.50 \\
\hline 5 & 1.35 & 1.49 & 1.03 & 1.59 & 2.42 & 1.13 & 1.03 & 1.04 & 1.03 & 1.03 \\
\hline 6 & 1.09 & 0.97 & 1.09 & 1.33 & 1.04 & 1.18 & 1.48 & 0.79 & 1.22 & 0.42 \\
\hline 7 & 2.23 & 1.57 & 1.54 & 1.22 & 1.37 & 1.08 & 2.26 & 1.14 & 0.94 & 1.31 \\
\hline 8 & 1.55 & 0.98 & 1.01 & 2.94 & 1.58 & 1.03 & 1.04 & 1.00 & 2.45 & 1.86 \\
\hline 9 & 1.92 & 1.04 & 1.30 & 1.26 & 1.30 & 0.94 & 1.58 & 1.52 & 1.21 & 2.33 \\
\hline 10 & 1.00 & 1.42 & 1.19 & 1.67 & 0.95 & 1.34 & 2.90 & 1.39 & 2.01 & 2.70 \\
\hline 11 & 2.31 & 1.27 & 1.67 & 2.75 & 1.31 & 1.30 & 1.61 & 0.80 & 1.24 & 1.53 \\
\hline 12 & 1.16 & 1.11 & 1.01 & 1.53 & 1.33 & 1.37 & 1.20 & 0.96 & 1.25 & 0.96 \\
\hline 13 & 1.24 & 1.04 & 1.05 & 1.17 & 1.04 & 1.04 & 1.03 & 1.31 & 1.00 & 1.01 \\
\hline 14 & 1.58 & 1.06 & 1.27 & 1.01 & 1.74 & 1.36 & 1.82 & 1.18 & 1.89 & 2.02 \\
\hline 15 & 1.41 & 1.21 & 0.97 & 1.68 & 1.18 & 1.08 & 1.68 & 1.06 & 1.18 & 1.38 \\
\hline 16 & 1.52 & 1.10 & 1.34 & 1.90 & 1.12 & 1.16 & 1.34 & 1.23 & 0.93 & 1.07 \\
\hline 17 & 1.08 & 1.38 & 1.15 & 0.71 & 1.30 & 1.26 & 1.42 & 1.21 & 1.01 & 1.59 \\
\hline 18 & 1.81 & 1.15 & 1.49 & 2.05 & 1.27 & 1.18 & 1.31 & 2.18 & 1.16 & 1.34 \\
\hline 19 & 1.52 & 0.97 & 0.94 & 0.77 & 1.11 & 1.15 & 1.34 & 0.83 & 1.11 & 1.20 \\
\hline 20 & 0.99 & 1.23 & 1.04 & 1.22 & 0.99 & 1.12 & 1.42 & 1.44 & 1.03 & 1.49 \\
\hline$\mu$ & 1.50 & 1.18 & 1.19 & 1.50 & 1.27 & 1.16 & 1.45 & 1.20 & 1.23 & 1.38 \\
\hline $\mathrm{COV}$ & $\mathbf{0 . 2 7}$ & $\mathbf{0 . 1 7}$ & 0.18 & $\mathbf{0 . 3 8}$ & 0.26 & 0.11 & $\mathbf{0 . 3 3}$ & $\mathbf{0 . 2 7}$ & 0.34 & $\mathbf{0 . 3 9}$ \\
\hline
\end{tabular}




\section{Cortantes de entrepiso}

El parámetro R se estima siguiendo el mismo procedimiento que para el caso de carga axial, la única diferencia es que ahora representa razones de cortantes de entrepiso. Solo se presentan las estadísticas para comportamiento inelástico, estas se dan en la Tabla 12. En comparación al caso de carga axial, la variación de $\mathrm{R}$ de un terremoto a otro y de un entrepiso a otro es mucho menor. La media y el COV, considerando ambos modelos, todos los entrepisos y todos los terremotos, son 1.08 y 0.12 , respectivamente. Esto indica que la respuesta crítica es muy cercana a la producida por las componentes principales. No se observan diferencias significativas en las estadísticas de $\mathrm{R}$ para comportamiento elástico e inelástico ni para dos y tres componentes. En resumen, el parámetro $\mathrm{R}$ varía considerablemente con el ángulo de incidencia de las componentes, y la respuesta crítica en términos de carga axial y cortante de entrepiso es mayor a la producida por las componentes principales, particularmente para comportamiento inelástico. Los valores de las medias de $\mathrm{R}$ y la incertidumbre en su estimación son mucho mayores para carga axial que para cortantes de entrepiso. El ángulo crítico es, en general, diferente para cada elemento considerado y para comportamiento elástico e inelástico. Se propone que la respuesta crítica se obtenga como 1.30 y 1.10 veces la respuesta producida por las componentes principales, para carga axial y cortantes de entrepiso, respectivamente.

Tabla 12. Valores máximos de R, cortantes de entrepiso, comportamiento elástico

\begin{tabular}{|c|c|c|c|c|c|c|c|c|c|c|c|c|}
\hline \multirow{2}{*}{ Sismo } & \multicolumn{3}{|c|}{ Modelo 1} & \multicolumn{9}{|c|}{ Modelo 2} \\
\hline & ENT 3 & ENT 2 & BASE & ENT 9 & ENT 8 & ENT 7 & ENT 6 & ENT 5 & ENT 4 & ENT 3 & ENT 2 & BASE \\
\hline 1 & 1.03 & 1.00 & 1.04 & 0.98 & 0.97 & 0.98 & 0.98 & 1.05 & 0.98 & 1.01 & 0.99 & 1.04 \\
\hline 2 & 1.03 & 0.97 & 0.95 & 1.25 & 1.22 & 1.17 & 1.14 & 1.11 & 1.08 & 1.08 & 1.13 & 1.08 \\
\hline 3 & 1.15 & 1.18 & 1.19 & 1.03 & 1.04 & 1.05 & 1.02 & 1.05 & 1.05 & 1.09 & 1.10 & 1.07 \\
\hline 4 & 1.08 & 0.98 & 1.01 & 0.93 & 0.86 & 0.93 & 0.92 & 0.89 & 0.98 & 0.98 & 1.07 & 0.98 \\
\hline 5 & 1.24 & 1.45 & 1.25 & 1.05 & 1.20 & 1.14 & 1.08 & 1.09 & 1.14 & 1.10 & 1.13 & 1.05 \\
\hline 6 & 1.04 & 1.04 & 1.02 & 1.19 & 1.18 & 1.19 & 1.14 & 1.08 & 1.09 & 1.10 & 1.11 & 1.14 \\
\hline 7 & 1.04 & 1.09 & 1.06 & 1.04 & 1.02 & 1.05 & 1.05 & 1.07 & 1.06 & 1.00 & 1.09 & 1.07 \\
\hline 8 & 1.03 & 1.01 & 1.01 & 1.11 & 1.15 & 1.20 & 1.16 & 1.11 & 1.10 & 1.08 & 1.13 & 1.10 \\
\hline 9 & 1.11 & 1.11 & 1.18 & 0.96 & 0.97 & 1.00 & 1.03 & 0.99 & 1.01 & 1.03 & 0.97 & 0.96 \\
\hline 10 & 1.02 & 1.03 & 1.08 & 1.52 & 1.39 & 1.61 & 1.43 & 1.45 & 1.53 & 1.79 & 1.34 & 1.66 \\
\hline 11 & 0.99 & 1.15 & 1.08 & 1.09 & 1.03 & 1.05 & 0.93 & 0.88 & 0.98 & 1.02 & 1.04 & 0.91 \\
\hline 12 & 1.06 & 1.06 & 1.17 & 0.98 & 0.99 & 1.08 & 1.09 & 1.08 & 1.07 & 1.09 & 1.13 & 1.06 \\
\hline 13 & 1.03 & 1.00 & 1.04 & 1.08 & 1.10 & 1.13 & 1.09 & 1.05 & 1.06 & 1.01 & 1.03 & 1.05 \\
\hline 14 & 1.16 & 1.09 & 1.11 & 1.33 & 1.27 & 1.18 & 1.20 & 1.33 & 1.10 & 1.11 & 1.11 & 1.10 \\
\hline 15 & 1.05 & 0.98 & 0.93 & 1.09 & 1.08 & 1.06 & 1.03 & 1.04 & 1.02 & 1.01 & 1.02 & 1.02 \\
\hline 16 & 1.01 & 1.03 & 1.05 & 1.09 & 1.09 & 1.09 & 1.09 & 1.03 & 1.08 & 1.03 & 1.10 & 1.10 \\
\hline 17 & 0.97 & 1.06 & 1.03 & 1.17 & 1.19 & 1.15 & 1.15 & 1.11 & 1.08 & 1.07 & 1.10 & 1.10 \\
\hline 18 & 1.05 & 1.05 & 1.03 & 1.07 & 1.03 & 1.05 & 1.04 & 1.07 & 1.03 & 1.06 & 1.11 & 1.09 \\
\hline 19 & 1.07 & 1.05 & 1.06 & 1.06 & 1.05 & 1.14 & 1.17 & 1.11 & 1.09 & 1.08 & 1.11 & 1.09 \\
\hline 20 & 1.02 & 1.03 & 1.02 & 1.03 & 1.05 & 1.02 & 1.05 & 1.01 & 1.01 & 0.99 & 0.98 & 1.02 \\
\hline$\mu$ & 1.06 & 1.07 & 1.07 & 1.10 & 1.09 & 1.11 & 1.09 & 1.08 & 1.08 & 1.09 & 1.09 & 1.08 \\
\hline \multirow[t]{2}{*}{$\mathrm{COV}$} & 0.06 & 0.10 & 0.08 & 0.13 & 0.11 & 0.12 & 0.10 & 0.12 & 0.11 & 0.16 & 0.07 & 0.13 \\
\hline & & & & \multicolumn{2}{|c|}{$\mu($ Todos $)=1.08$} & \multicolumn{4}{|c|}{$\operatorname{COV}($ Todos $)=0.12$} & & & \\
\hline
\end{tabular}




\section{CONCLUSIONES}

En este trabajo se estudió la precisión de las reglas del 30\% y la de la Raíz Cuadrada de la Suma de los Cuadrados (SRSS) comúnmente consideradas en los códigos para combinar los efectos individuales de las componentes sísmicas, así como la influencia de la correlación de las componentes, y la de sus correspondientes efectos individuales, en dicha precisión. Se consideraron edificios de acero con marcos resistentes a momento perimetrales modelados como sistemas de varios grados de libertad (SVGL). La precisión de las reglas se estudió para comportamiento elástico e inelástico, parámetros de respuesta colineales y no colineales, parámetros de respuesta individuales y múltiples, y para dos y tres componentes de los registros sísmicos. Además, se estimaron las respuestas sísmicas para varios ángulos de incidencia de las componentes horizontales a fin de encontrar el ángulo crítico de éstas. Para ello, se usaron algunos modelos estructurales del proyecto de la SAC, los cuales se excitaron por veinte registros sísmicos en el dominio del tiempo. Los resultados de este estudio indican que, en general, ambas reglas de combinación subestiman la carga axial alrededor de $15 \%$ y la incertidumbre asociada a dicha subestimación (COV) es alrededor de 30\%. Para cortante, ambas reglas sobreestiman la respuesta combinada alrededor de $10 \%$. La incertidumbre en la estimación de la precisión de las reglas es mucho menor para cortante que para carga axial. Las reglas son ligeramente más precisas cuando los modelos se excitan por las tres componentes de los terremotos que cuando se excitan solo por las dos componentes horizontales. Cuando las reglas se aplican a parámetros de respuesta múltiples, la respuesta combinada se subestima alrededor del 15\%. Para este caso, las estadísticas son esencialmente las mismas para comportamiento elástico e inelástico y para dos y tres componentes. Sin embargo, el nivel de incertidumbre se incrementa cuando se considera comportamiento inelástico. Se muestra también que los efectos de las componentes individuales pueden estar altamente correlacionados, no solo para componentes normales sino también para componentes no correlacionadas (principales). Las reglas no siempre son precisas para valores pequeños de coeficientes de correlación y valores altos de éstos no están necesariamente relacionados a una estimación imprecisa de la respuesta combinada. En general, la precisión de las reglas de combinación depende del grado de correlación de las componentes de los terremotos y de sus correspondientes efectos individuales, del parámetro de respuesta al cual se aplican, de la localización del elemento considerado y del nivel de deformación estructural. Los códigos deben ser más específicos con respecto a la aplicación de las reglas de combinación anteriormente mencionadas. La respuesta crítica ocurre, en general, para un ángulo de incidencia diferente al de las componentes normales o principales, y el ángulo crítico varía de un sismo a otro y de un elemento a otro. La respuesta crítica se puede estimar como 1.30 y 1.10 veces la respuesta producida por las componentes principales, para carga axial y cortantes de entrepiso, respectivamente, para el sistema estructural considerado.

\section{AGRADECIMIENTOS}

Se agradecen las críticas de los revisores anónimos, las cuales enriquecieron la calidad de este trabajo. Esta investigación se realizó con el apoyo otorgado por la Universidad Autónoma de Sinaloa dentro del proyecto PROFAPI-2013/157. El primer autor agradece al Consejo Nacional de Ciencia y Tecnología (CONACYT) la beca otorgada durante sus estudios de posgrado.

\section{REFERENCIAS}

AISC (2005), "Specification for Structural Steel Buildings", American Institute of Steel Construction, Chicago, IL. 
Beyer, K. y Bommer J.J. (2007), "Selection and scaling of real accelerograms for bi-directional loading: a review of current practice and code provisions", Journal of Earthquake Engineering,11(1), 13-45.

Bisadi, V. y Head, M. (2010), "Orthogonal effects in nonlinear analysis of bridges subjected to multicomponent earthquake excitation”, Structures Congress of the American Society of Civil Engineers, Section Bridges, pp. 204-215.

Bojorquez, E., Reyes-Salazar A., Terán-Gilmore A. y Ruiz, S.E. (2010), "Energy-based damage index for steel structures", Steel and Composite Structures An International Journal, (10) 4, 331-348.

Building Officials \& Code Administration International (1993), 12th Edition, National Building Code.

Federal Emergency Management Agency (2000), State of the Art Report on Systems Performance of Steel Moment Frames Subjected to Earthquake Ground Shaking, SAC Steel Project, Report FEMA 355C.

Heredia-Zavoni E. y Machicao Barrionuevo R. (2004a), "Response to orthogonal components of ground motion and assessment of percentage combination rules, Earthquake Engineering and Structural Dynamics, 33, 271-284.

Heredia Zavoni E. y Machicao Barrionuevo R. (2004b), "Respuesta a componentes ortogonales de excitación sísmica y análisis de las reglas de combinación porcentual", XIV Congreso Nacional de Ingeniería Estructural, Acapulco Gro.

International Code Council (2009), International Building Code (IBC), Falls Church, VA.

Kondo, K. y Atluri, S.N. (1987), "Large deformation elasto-plastic analysis of frames under nonconservative loading using explicitly derived tangent stiffness based on assumed stress", Computational Mechanics2(1), 1-25.

Lopez, O.A., Chopra, A.K. y Hernandez, J.J. (2000) "Critical response of structures to multi-component earthquake excitation”, Earthquake Engineering and Structural Dynamics, 29, 1759-1778.

Lopez, O.A., Hernandez J.J., Bonilla, R. y Fernandez A. (2006), "Response spectra for multi-component structural analysis", Earthquake Spectra, 22(1), 85-113.

Mackie, K.R. y Cronin K.J. (2011), "Response sensitivity of highways bridges to randomly oriented multi-component earthquake excitation", Journal of Earthquake Engineering, 15(6), 850-876.

McKenna, F. y Feneves, G. L. (2009). “Open system for earthquake engineering simulation”, Pacific earthquake engineering research center, version 2.1.0.

Newmark, N.M. (1975) "Seismic design criteria for structures and facilities, Trans-Alaska pipeline system", Proceedings of the U.S. National Conference on Earthquake Engineering, pp. 94-103.

Newmark, N.M. y Hall, W.J. (1982), Earthquake Spectra and Design, Monograph Series, Earthquake Engineering Institute, Berkeley CA, USA.

Penzien, J. y Watabe, M. (1975), "Characteristics of 3-Dimensional earthquake ground motions", Earthquake Engineering and Structural Dynamics3, 365-373.

Reglamento de construcciones del Distrito Federal (2004), Normas Técnicas Complementarias de Diseño por Sismo, Gaceta Oficial del Distrito Federal.

Reyes-Salazar, A. (1997) "Inelastic Seismic Response and Ductility Evaluation of Steel Frames with Fully, Partially Restrained and Composite Connections", PhD. thesis Department of Civil Engineering and Engineering Mechanics, University of Arizona, Tucson, Arizona. 
Reyes-Salazar, A. y Haldar, A. (1999), "Nonlinear Seismic Response of Steel Structures with Semi-rigid and Composite Connections", Journal of Constructional Steel Research, 51, 37-59.

Reyes-Salazar, A., Haldar, A. y Romero-Lopez, M.R. (2000), "Force reduction factor for SDOF and MDOF”, Joint Specialty Conference on Probabilistic Mechanics and Structural, ASCE, Paper 063.

Reyes-Salazar, A. y Haldar, A. (2000), "Dissipation of Energy in Steel Frames With PR Connections", Structural Engineering and Mechanics, an International Journal, 9(3), 241-256.

Reyes-Salazar, A. y Haldar, A. (2001a) "Energy Dissipation at PR Frames Under Seismic Loading”, Journal of Structural Engineering ASCE, 127(5), 588-593.

Reyes-Salazar, A. y Haldar, A. (2001b), “Seismic Response and Energy Dissipation in Partially Restrained and Fully Restrained Steel Frames: An Analytical Study", Steel \& Composite Structures, An International Journal, 1(4), 459-480.

Reyes-Salazar, A., Juarez-Duarte, J.A., López-Barraza, A. y Velázquez-Dimas, J.I. (2004), “Combined effect of the horizontal components of earthquakes for moment resisting steel frames", Steel \& Composite Structures An International Journal,4(3), 89-209.

Reyes-Salazar, A., López-Barraza, A., López-López, L.A. y Haldar, A. (2008), "Multiple-Components Seismic Response Analysis-A Critical Review", Journal of Earthquake Engineering,12(5), 779799.

Rigato, A. B. y Medina, R. A. (2007), "Influence of angle of incidence on seismic demands for inelastic single-storey structures subjected to bi-directional ground motions", EngineeringStructures 29(10), 2593-2601.

Rosenblueth, E. (1980), Design of Earthquake Resistance Structures, Pentech Press Ltd.

Rosenblueth, E. y Contreras, H. (1977), “Approximate Design for multi-component Earthquakes”, Journal of Engineering Mechanics Division ASCE, 103, 895-911.

Salmon, C.G., Johnson, J.E. y Malhas, F.A. (2009), Steel Structures Design and Behavior, Fifth Edition, Pearson, Prentice Hall, New Jersey.

Smeby, W. y Der Kiureghian, A. (1985). Modal combination rules for multi-component earthquake excitation. Earthquake Engineering and Structural Dynamics 1985, 13, 1-12.

Uniform Building Code (1994), Structural Engineering Design Provisions Vol. 2, International Conference of Building Officials.

Valdés González J. y Ordaz Schroeder M. (2008), “Análisis de efectos sísmicos ortogonales horizontales en terreno blando", Revista de Ingeniería Sísmica, No 79, pp91-111.

Wilson, E.L., Suharwardy, I. y Habibullah, A. (1995), "A Clarification of the orthogonal effects in a threedimensional seismic analysis”, Earthquake Spectra,11(4), 659-666. 